Electronic Supporting Information

\title{
Reusable Ruthenium Microspheres Derived from Chitin for Highly Efficient and Selective Hydroboration of Imines
}

Zirui Lin ${ }^{\dagger}$, Jie Zou ${ }^{\ddagger}$, Shufen $\mathrm{Li}^{\ddagger}$, Chi Zhang ${ }^{\S}$, Feng, Xie, ${ }^{\dagger}$ Bin Li, ${ }^{*}{ }^{\dagger}$ Dongdong Ye*,*

'School of Biotechnology and Health Sciences, Wuyi University, Jiangmen 529020,

China

:School of Textile Materials and Engineering, Wuyi University, Jiangmen 529020 ,

China.

§School of Applied Physics and Materials, Wuyi University, Jiangmen 529020, China

Table of Contents

S2 General remarks

S3 Preparation of the PCRuMs catalyst

S4 Characterization of PCRuMs catalysts

S7 General procedures for PCRuMs catalyzed hydroboration reactions

S8 Characterization data of substrates

S18 1H and 13C NMR Spectra 


\section{General remarks}

All reagents were obtained from commercial sources and used as received. Crab shell was provided by Golden-Shell Biochemical Co. Ltd (Zhejiang, China). Ruthenium trichloride was purchased from Energy Chemical Regents (Shanghai, China). Acetone, potassium hydroxide, sodium hydroxide, urea, Tween 85, isooctane, sodium hypochlorite, and absolute ethyl alcohol were purchased from Guangzhou Chemical Reagents (Guangzhou, China). Dimethyl carbonate (anhydrous) were used as received. Technical grade petroleum ether $\left(40-60^{\circ} \mathrm{C}\right.$ bp. $)$ and ethyl acetate were used for chromatography column.

SEM images of CMs and PCRuMs were taken with an FE-SEM (Sigma 500, Zeiss, Germany) operating at an acceleration voltage of $5 \mathrm{kV}$. Optical microscope picture was recorded on a polarizing microscope (DM2700P, Leica, Germany). TEM images of the super-thin sliced PCRuMs were taken with a transmission electron microscopy (JEM-2100, JEOL, Japan) operating at an accelerating voltage of $200 \mathrm{kV}$. The partial carbonization process on the chitin/ $\mathrm{Ru}^{3+}$ microspheres was carried out on a thermogravimetric analysis system (Q50, TA, America) at a heating rate of $10{ }^{\circ} \mathrm{C}$ $\min ^{-1}$ from 25 to $250{ }^{\circ} \mathrm{C}$, followed by a constant temperature at $250{ }^{\circ} \mathrm{C}$ for $4 \mathrm{~h}$ under the nitrogen atmosphere. The Brunauer-Emmett-Teller (BET) surface areas were measured and calculated by the nitrogen sorption-desorption isotherms using an adsorption analyzer (ASAP 2020, Micromeritics, USA). 2D Raman imaging was performed using a laser confocal Raman imaging microscope (inVia, Renishaw, UK). The wavelength of the excitation laser was $532 \mathrm{~nm}$. Raman maps (scan range, $50 \mu \mathrm{m}$ $\times 50 \mu \mathrm{m}$ ) were collected using a spatial resolution of $200 \mathrm{~nm}$. The direct classical least squares (DCLS) method developed by accessory software was applied for imaging the chitin and the amorphous carbon domains. X-ray photoelectron spectra was conducted on an X-ray photoelectron spectrometer (ESCALAB250Xi, Thermo Fisher Scientific, America) with monochromatic Al target test, and full spectrum passing energy $100 \mathrm{eV}$.

${ }^{1} \mathrm{H}$ NMR spectra were recorded in $\mathrm{CDCl}_{3}$ at ambient temperature on Bruker AVANCE I 500 spectrometers at $500.1 \mathrm{MHz}$, using the solvent as internal standard (7.26 ppm). ${ }^{13} \mathrm{C}$ NMR spectra were obtained at $125 \mathrm{MHz}$ and referenced to the internal solvent signals (central peak is $77.2 \mathrm{ppm}$ ). Chemical shift $(\delta)$ and coupling constants $(J)$ are given in ppm and in Hz, respectively. The peak patterns are indicated as follows: s, singlet; d, doublet; t, triplet; q, quartet; m, multiplet, and br. for broad.

GC analyses were performed with GC-7890A (Agilent) equipped with a 30-m capillary column (HP-5ms, fused silica capillary column, $30 \mathrm{M}^{*} 0.25 \mathrm{~mm} * 0.25 \mathrm{~mm}$ film thickness), was used with $\mathrm{N}_{2} /$ air as vector gas. GCMS were measured by GCMS-7890A-5975C (Agilent) with GC-7890A equipped with a 30-m capillary column (HP-5ms, fused silica capillary column, $30 \mathrm{M}^{*} 0.25 \mathrm{~mm} * 0.25 \mathrm{~mm}$ film thickness), was used with helium as vector gas. HRMS were measured by MAT 95XP (Termol) (LCMS-IT-TOF). 


\section{Chitin microspheres (CMs) preparation.}

Crab shells were crushed into powders, and then $1 \mathrm{~mol} / \mathrm{L} \mathrm{NaOH}, 7$ wt $\% \mathrm{HCl}$ and $0.3 w t \% \mathrm{NaClO}_{2}$ aqueous solution was used in turn to remove impurities including proteins, minerals and pigments in crab powers. Then, the desired amount of above chitin powers was dispersed in $20 w t \% \mathrm{KOH} / 4 w t \%$ urea aqueous solution, while stirring at $-30{ }^{\circ} \mathrm{C}$ for $2 \mathrm{~h}$, to obtain a transparent and viscous chitin solution. Subsequently, $11 \mathrm{~g}$ of Span 85 and $250 \mathrm{~g}$ of isooctane were mixed and stirred at 896 rpm for $30 \mathrm{~min}$. Then, chitin solution was poured to the suspension at $0{ }^{\circ} \mathrm{C}$ and stirred for $40 \mathrm{~min}$. Subsequently, the mixture containing $7.5 \mathrm{~g}$ of isooctane was then dropped into the emulsion for $30 \mathrm{~min}$ to form spherical chitin droplets wrapped in an oil phase. Finally, the mixture was rapidly heated to $60^{\circ} \mathrm{C}$ to gel the chitin solution and induce the formation of the chitin microspheres. After washing out the oil phase and the residual solvent, chitin microspheres with nanofibers structure, coded as CMs, were obtained after supercritical drying.

\section{Partially carbonized chitin/Ru microspheres (PCRuMs) preparation.}

$500 \mathrm{mg}$ of the above chitin microspheres and $\mathrm{RuCl}_{3}[8.73 \mathrm{mg}, 0.0421 \mathrm{mmol}$ for 1.39 $\mathrm{wt} \% \mathrm{Ru} / \mathrm{chitin}]$ were mixed in $50 \mathrm{~mL}$ of acetone, while stirring for $5 \mathrm{~h}$. The obtained chitin $/ \mathrm{Ru}^{3+}$ microspheres were successively separated and supercritical dried. Then, the composite microspheres were calcinated at $250{ }^{\circ} \mathrm{C}$ under argon atmosphere for $4 \mathrm{~h}$ to fabricate the partially carbonized chitin/Ru microspheres. 


\section{Characterization of PCRuMs catalysts}
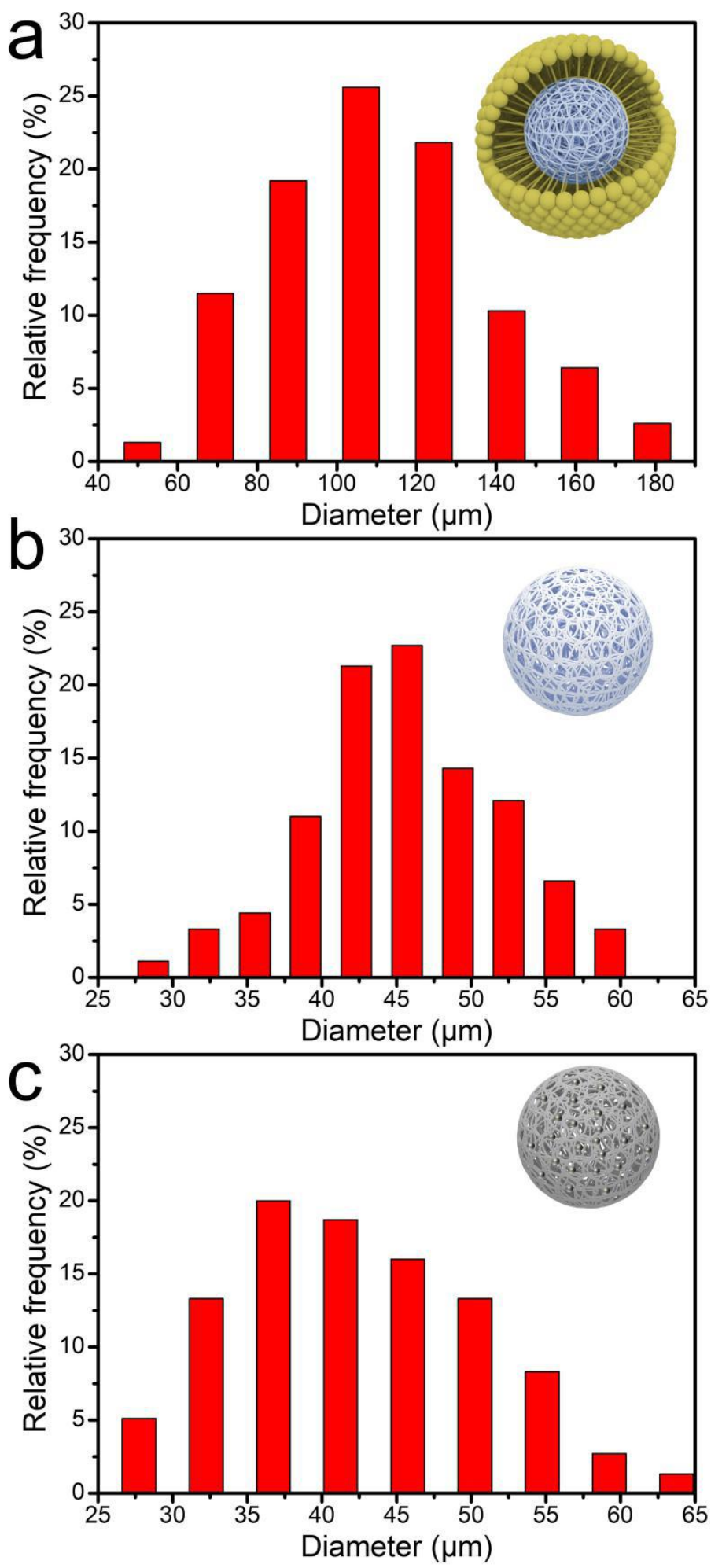

Figure S1. Size distribution of the emulsified spherical chitin solution (ECS) (a), regenerated chition microspheres (RCHs) (b) and partially carbonized chitin/Ru microspheres (PCRMs) (c), demonstrating a variation of gradually decreased diameter of microspheres. 


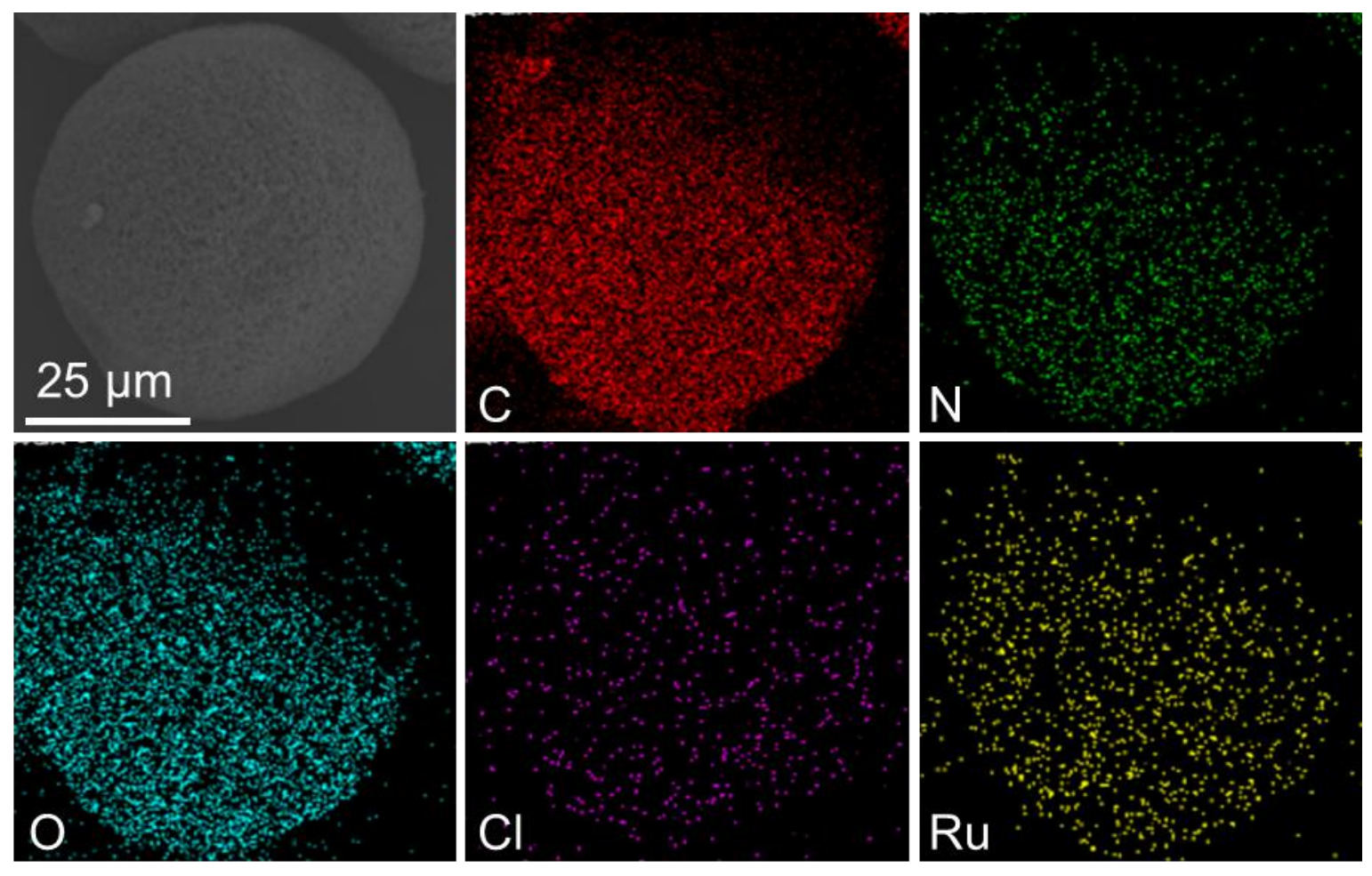

Figure S2. SEM image of the chitin/ $\mathrm{Ru}^{3+}$ composite microspheres and the corresponding elemental mappings of $\mathrm{C}, \mathrm{N}, \mathrm{O}, \mathrm{Cl}$ and $\mathrm{Ru}$. 

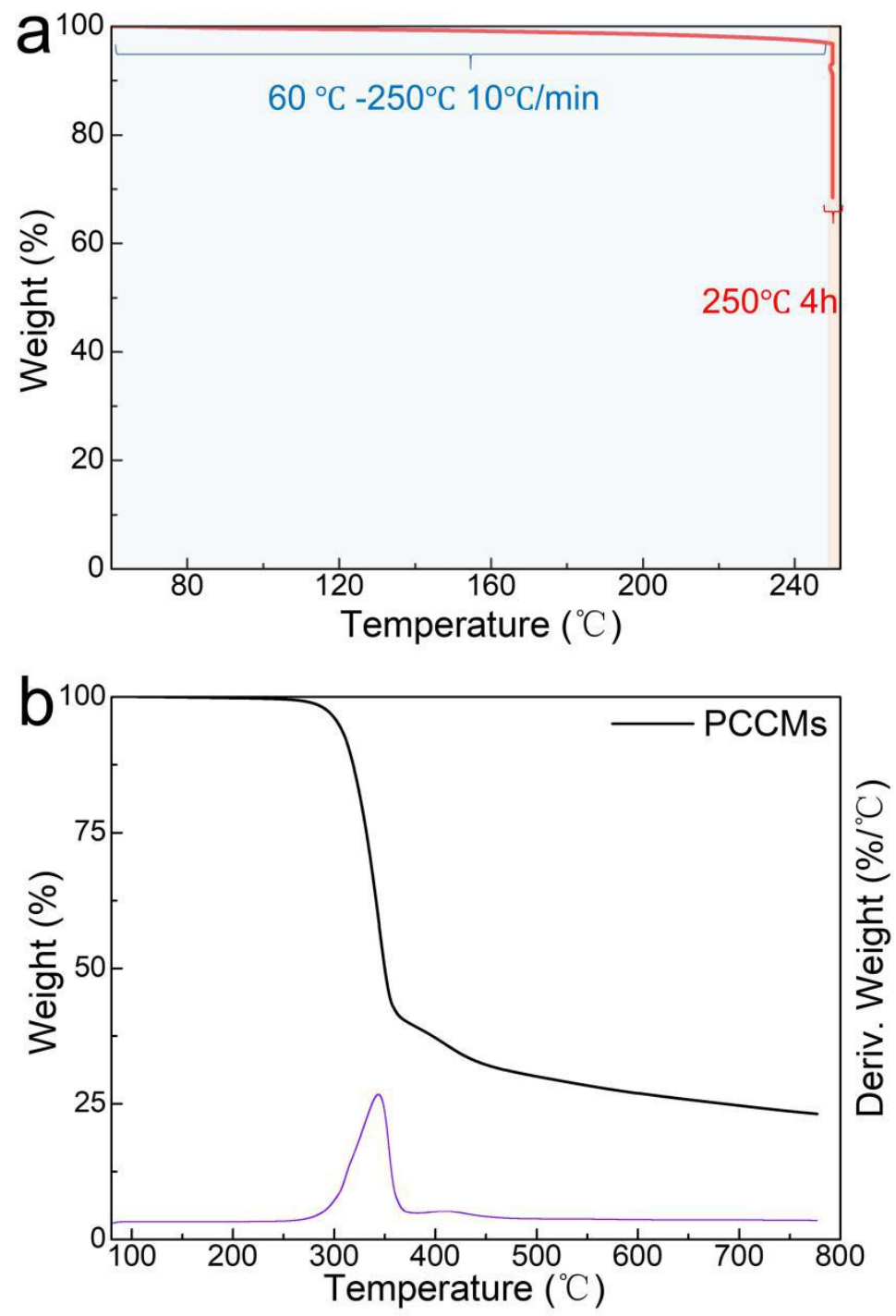

Figure S3. Monitoring the partially carbonized process of the chitin/ $\mathrm{Ru}^{3+}$ microspheres with TG instrument. TG spectra of the fabrication process of the PCRMs(a) and TG spectra of the PCRMs (b), demonstrating the feasibility of carbonization at lower temperatures. 

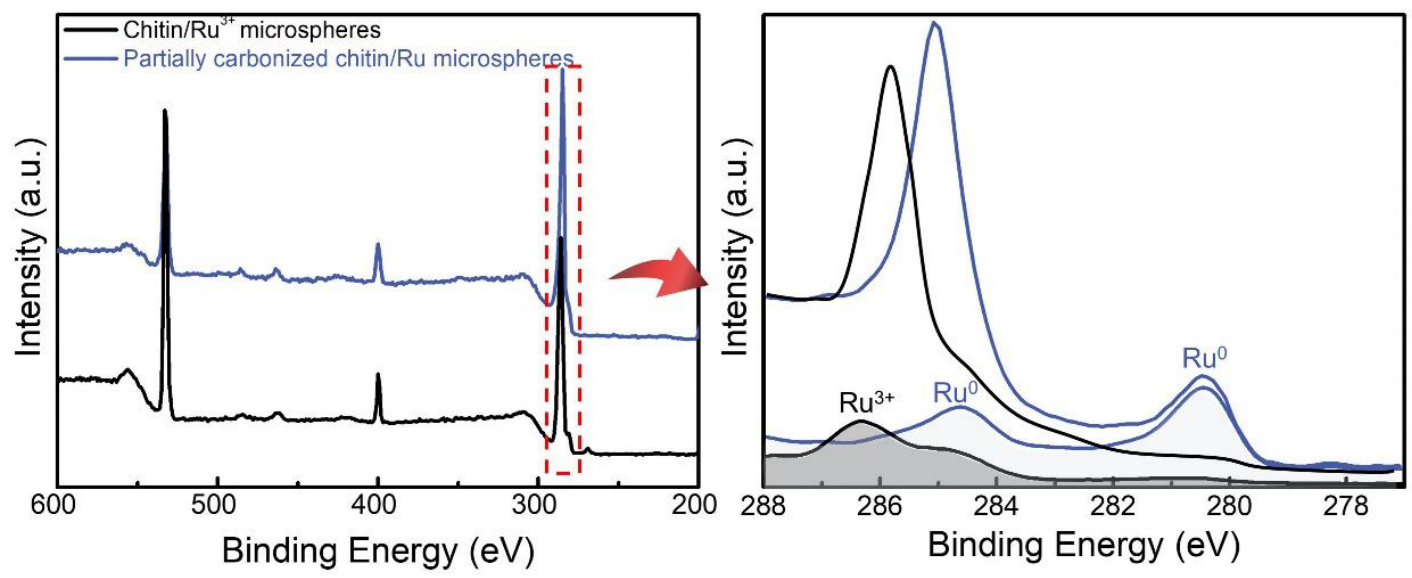

Figure S4. XPS spectra of the chitin/ $\mathrm{Ru}^{3+}$ microspheres and partially carbonized chitin/Ru microspheres. Apparenyly, the binding energy peaks of $\mathrm{Ru}^{3+} 3 \mathrm{~d}_{3 / 2}$ (focusing on $286.3 \mathrm{eV}$ ) in chitin/ $\mathrm{Ru}^{3+}$ microspheres changed to the $284.4 \mathrm{eV}$ (base peak of $\mathrm{Ru}(0)$ $3 \mathrm{~d}_{3 / 2}$ ) and $280.4 \mathrm{eV}$ (another base peak of $\mathrm{Ru}(0) 3 \mathrm{~d}_{5 / 2}$ ) in partially carbonized chitin/Ru microspheres, confirming the feasibility of our rational design in constructing the $\mathrm{Ru}$ NPs on biomass polysaccharides from chitin/ $\mathrm{Ru}^{3+}$ with a partial carbonization process. 


\section{General procedure for PCRuMs catalyzed hydroboration of imines}

PCRuMs (0.0007 mmol, $10 \mathrm{mg})$, imine (0.5 mmol), HBpin (2.0 mmol, $288 \mu \mathrm{L})$, and DMC ( $2 \mathrm{~mL})$ were introduced in tube under air, equipped with magnetic stirring bar and was stirred at room temperature. After $2 \mathrm{~h}$, the conversion of the reaction was analyzed by gas chromatography. The solvent was then evaporated under vacuum and the desired product was purified by using a silica gel chromatography column and a mixture of petrol ether/ethyl acetate as eluent. 


\section{Characterization data of substrates}

N-benzylaniline (2a) ${ }^{1}$<smiles>c1ccc(CNc2ccccc2)cc1</smiles>

Light yellow oil, yield $=80 \%, 73 \mathrm{mg}$.

${ }^{1} \mathrm{H}$ NMR $\left(500 \mathrm{MHz}, \mathrm{CDCl}_{3}\right): \delta=7.46-7.23(\mathrm{~m}, 5 \mathrm{H}), 7.27-7.24(\mathrm{~m}, 2 \mathrm{H}), 6.81-6.78(\mathrm{~m}, 1 \mathrm{H})$, 6.72-6.69 (m, 2H), 4.39 (s, 2H), 4.08 (brs, $1 \mathrm{H})$.

${ }^{13} \mathrm{C}$ NMR $\left(125 \mathrm{MHz}, \mathrm{CDCl}_{3}\right): \delta=148.2,139.5,129.4,128.8,127.7,127.4,117.7,113.0$, 48.4 .

N-(4-methylbenzyl)aniline (2b) ${ }^{1}$<smiles>Cc1ccc(CNc2ccccc2)cc1</smiles>

Light yellow oil, yield $=86 \%, 85 \mathrm{mg}$.

${ }^{1} \mathrm{H}$ NMR $\left(500 \mathrm{MHz}, \mathrm{CDCl}_{3}\right): \delta=7.37(\mathrm{~d}, 2 \mathrm{H}, J=8.0 \mathrm{~Hz}), 7.30-7.26(\mathrm{~m}, 4 \mathrm{H}), 6.85-6.81(\mathrm{~m}$, $1 \mathrm{H}), 6.75-6.73(\mathrm{~m}, 2 \mathrm{H}), 4.38(\mathrm{~s}, 2 \mathrm{H}), 4.05$ (brs, $1 \mathrm{H}), 2.46(\mathrm{~s}, 3 \mathrm{H})$.

${ }^{13} \mathrm{C}$ NMR (125 MHz, $\left.\mathrm{CDCl}_{3}\right): \delta=148.3,137.0,136.5,129.4,129.3,127.6,117.6,113.0,48.2$, 21.2 .

N-(3-methylbenzyl)aniline (2c) $)^{2}$<smiles>Cc1cccc(CNc2ccccc2)c1</smiles>

Light yellow oil, yield $=60 \%, 59 \mathrm{mg}$.

${ }^{1} \mathrm{H}$ NMR (500 MHz, $\left.\mathrm{CDCl}_{3}\right): \delta=7.36-7.28(\mathrm{~m}, 5 \mathrm{H}), 7.21(\mathrm{~d}, 1 \mathrm{H}, J=7.5 \mathrm{~Hz}), 6.84(\mathrm{~d}, 1 \mathrm{H}, J=$ $7.5 \mathrm{~Hz}), 6.74$ (d, 2H, $J=8.5 \mathrm{~Hz}$ ), 4.38 (s, 2H), 4.08 (brs, 1H), 2.47 (s, 3H).

${ }^{13} \mathrm{C}$ NMR $\left(125 \mathrm{MHz}, \mathrm{CDCl}_{3}\right): \delta=148.3,139.5,138.4,129.4,128.7,128.4,128.1,124.7$, 117.6, 112.9, 48.4, 21.6 .

\section{N-(2-methylbenzyl)aniline (2d) ${ }^{3}$}<smiles>Cc1ccccc1CNc1ccccc1</smiles>

Light yellow oil, yield $=71 \%, 70 \mathrm{mg}$. 
${ }^{1} \mathrm{H}$ NMR (500 MHz, $\left.\mathrm{CDCl}_{3}\right): \delta=7.45(\mathrm{~d}, 1 \mathrm{H}, J=7.0 \mathrm{~Hz}), 7.34-7.28(\mathrm{~m}, 5 \mathrm{H}), 6.86(\mathrm{t}, 1 \mathrm{H}, J=$ $7.5 \mathrm{~Hz}), 6.76-6.74$ (m, 2H), 4.38 (s, 2H), 3.93 (brs, 1H), 2.50 (s, 3H).

${ }^{13} \mathrm{C}$ NMR $\left(125 \mathrm{MHz}, \mathrm{CDCl}_{3}\right): \delta=148.4,137.1,136.4,130.5,129.4,128.4,127.5,126.3$, $117.6,112.8,46.5,19.1$.

\section{N-(4-methoxybenzyl)aniline (2e) $)^{1}$}<smiles>COc1ccc(CNc2ccccc2)cc1</smiles>

Light yellow oil, yield $=75 \%, 80 \mathrm{mg}$.

${ }^{1} \mathrm{H}$ NMR $\left(500 \mathrm{MHz}, \mathrm{CDCl}_{3}\right): \delta=7.36(\mathrm{~d}, 2 \mathrm{H}, J=8.5 \mathrm{~Hz}), 7.27-7.24(\mathrm{~m}, 2 \mathrm{H}), 6.95(\mathrm{~d}, 2 \mathrm{H}, J=$ $9.0 \mathrm{~Hz}), 6.79(\mathrm{t}, 1 \mathrm{H}, J=7.5 \mathrm{~Hz}), 6.70(\mathrm{~d}, 2 \mathrm{H}, J=8.0 \mathrm{~Hz}), 4.31$ (s, 2H), 4.01 (brs, 1H), 3.87 (s, $3 \mathrm{H})$.

${ }^{13} \mathrm{C} \mathrm{NMR}\left(125 \mathrm{MHz}, \mathrm{CDCl}_{3}\right): \delta=158.9,148.3,131.5,129.4,128.9,117.6,114.1,112.9,55.4$, 47.9 .

N-(3-methoxybenzyl)aniline (2f)<smiles>COc1cccc(CNc2ccccc2)c1</smiles>

Light yellow oil, yield $=73 \%, 78 \mathrm{mg}$.

${ }^{1} \mathrm{H}$ NMR $\left(500 \mathrm{MHz}, \mathrm{CDCl}_{3}\right): \delta=7.35(\mathrm{t}, 1 \mathrm{H}, J=8.0 \mathrm{~Hz}), 7.29-7.26(\mathrm{~m}, 2 \mathrm{H}), 7.06-7.03(\mathrm{~m}$, 2H), 6.92-6.90 (m, 1H), $6.82(\mathrm{t}, 1 \mathrm{H}, J=7.5 \mathrm{~Hz}), 6.71(\mathrm{~d}, 2 \mathrm{H}, J=8.0 \mathrm{~Hz}), 4.38(\mathrm{~s}, 2 \mathrm{H}), 4.13$ (brs, 1H), 3.87 (s, 3H).

${ }^{13} \mathrm{C}$ NMR $\left(125 \mathrm{MHz}, \mathrm{CDCl}_{3}\right): \delta=160.0,148.2,141.3,129.7,129.4,119.8,117.6,113.1$, $112.9,112.7,55.3,48.3$.

\section{N-(2-methoxybenzyl)aniline (2g)}<smiles>COc1ccccc1CNc1ccccc1</smiles>

Light yellow solid, yield $=74 \%, 79 \mathrm{mg}$.

${ }^{1} \mathrm{H}$ NMR $\left(500 \mathrm{MHz}, \mathrm{CDCl}_{3}\right): \delta=7.42(\mathrm{~d}, 1 \mathrm{H}, J=7.5 \mathrm{~Hz}), 7.38-7.34(\mathrm{~m}, 1 \mathrm{H}), 7.30-7.27(\mathrm{~m}$, 2H), 7.04-6.98 (m, 2H), $6.82(\mathrm{t}, 1 \mathrm{H}, J=7.5 \mathrm{~Hz}), 6.76(\mathrm{~d}, 2 \mathrm{H}, J=8.0 \mathrm{~Hz}), 4.45(\mathrm{~s}, 2 \mathrm{H}), 4.20$ (brs, $1 \mathrm{H}), 3.95(\mathrm{~s}, 3 \mathrm{H})$.

${ }^{13} \mathrm{C}$ NMR $\left(125 \mathrm{MHz}, \mathrm{CDCl}_{3}\right): \delta=157.5,148.5,129.3,128.9,128.4,127.4,120.6,117.4$, $113.1,110.3,55.4,43.5$.

\section{N-(4-bromobenzyl)aniline (2h) ${ }^{2}$}


(N)

Brown solid, yield $=77 \%, 101 \mathrm{mg}$.

${ }^{1} \mathrm{H}$ NMR $\left(500 \mathrm{MHz}, \mathrm{CDCl}_{3}\right): \delta=7.54-7.52(\mathrm{~m}, 2 \mathrm{H}), 7.32-7.25(\mathrm{~m}, 4 \mathrm{H}), 6.82(\mathrm{t}, 1 \mathrm{H}, J=7.0$ $\mathrm{Hz})$, 6.69-6.67 (m, 2H), 4.34 (s, 2H), 4.06 (brs, 1H).

${ }^{13} \mathrm{C}$ NMR $\left(125 \mathrm{MHz}, \mathrm{CDCl}_{3}\right): \delta=147.9,138.6,131.8,129.4,129.1,121.0,117.9,113.0$, 47.7 .

\section{N-(4-(trifluoromethyl)benzyl)aniline (2i) ${ }^{2}$}<smiles>FC(F)(F)c1ccc(CNc2ccccc2)cc1</smiles>

Colorless solid, yield $=80 \%, 100 \mathrm{mg}$.

${ }^{1} \mathrm{H}$ NMR (500 MHz, $\mathrm{CDCl}_{3}$ ): $\delta=7.67(\mathrm{~d}, 2 \mathrm{H}, J=8.0 \mathrm{~Hz}$ ), 7.55 (d, 2H, $J=8.0 \mathrm{~Hz}$ ), 7.28-7.25 (m, 2H), 6.83 (t, 1H, $J=7.5 \mathrm{~Hz}), 6.69-6.68(\mathrm{~m}, 2 \mathrm{H}), 4.47$ (s, 2H), 4.20 (brs, $1 \mathrm{H})$.

${ }^{13} \mathrm{C}$ NMR $\left(125 \mathrm{MHz}, \mathrm{CDCl}_{3}\right): \delta=147.8,143.9,129.6\left(J_{\mathrm{CF}}=32.0 \mathrm{~Hz}\right), 129.5,127.6,125.7$ $\left(J_{\mathrm{CF}}=3.6 \mathrm{~Hz}\right), 125.5\left(J_{\mathrm{CF}}=270.6 \mathrm{~Hz}\right), 118.1,113.0,47.8$.

${ }^{19} \mathrm{~F} \mathrm{NMR}\left(376 \mathrm{MHz}, \mathrm{CDCl}_{3}\right): \delta=-183.0 \mathrm{~Hz}$.

\section{N-benzyl-4-methylaniline $(2 \mathrm{j})^{2}$}<smiles>Cc1ccc(NCc2ccccc2)cc1</smiles>

Light yellow oil, yield $=94 \%, 93 \mathrm{mg}$.

${ }^{1} \mathrm{H}$ NMR $\left(500 \mathrm{MHz}, \mathrm{CDCl}_{3}\right): \delta=7.48-7.36(\mathrm{~m}, 5 \mathrm{H}), 7.09(\mathrm{~d}, 2 \mathrm{H}, J=7.5 \mathrm{~Hz}), 6.66(\mathrm{~d}, 2 \mathrm{H}, J=$ $7.5 \mathrm{~Hz}), 4.40$ (s, 2H), 3.78 (brs, $1 \mathrm{H}), 2.35$ (s, 3H).

${ }^{13} \mathrm{C} \mathrm{NMR}\left(125 \mathrm{MHz}, \mathrm{CDCl}_{3}\right): \delta=146.0,139.8,129.9,128.7,127.6,127.3,126.9,113.1,48.7$, 20.5 .

\section{N-benzyl-4-methoxyaniline (2k) ${ }^{3}$}<smiles>COc1ccc(NCc2ccccc2)cc1</smiles>

Brown oil, yield $=85 \%, 91 \mathrm{mg}$.

${ }^{1} \mathrm{H}$ NMR (500 MHz, $\mathrm{CDCl}_{3}$ ): $\delta=7.47-7.35(\mathrm{~m}, 5 \mathrm{H}), 6.87(\mathrm{~d}, 2 \mathrm{H}, J=8.5 \mathrm{~Hz}), 6.68(\mathrm{~d}, 2 \mathrm{H}, J=$ $9.0 \mathrm{~Hz}), 4.36$ (s, 2H), 3.88 (brs, 1H), $3.82(\mathrm{~s}, 3 \mathrm{H})$. 
${ }^{13} \mathrm{C} \mathrm{NMR}\left(125 \mathrm{MHz}, \mathrm{CDCl}_{3}\right): \delta=152.2,142.5,139.8,128.7,127.6,127.2,114.9,114.2,55.8$, 49.2 .

\section{N-benzyl-4-bromoaniline (2I) ${ }^{2}$}<smiles>Brc1ccc(NCc2ccccc2)cc1</smiles>

Light yellow oil, yield $=80 \%, 105 \mathrm{mg}$.

${ }^{1} \mathrm{H}$ NMR (500 MHz, $\mathrm{CDCl}_{3}$ ): $\delta=7.45-7.35(\mathrm{~m}, 5 \mathrm{H}), 7.31(\mathrm{~d}, 2 \mathrm{H}, J=8.5 \mathrm{~Hz}), 6.55(\mathrm{~d}, 2 \mathrm{H}, J=$ $9.0 \mathrm{~Hz}), 4.35$ (s, 2H), 4.13 (brs, 1H).

${ }^{13} \mathrm{C}$ NMR $\left(125 \mathrm{MHz}, \mathrm{CDCl}_{3}\right): \delta=147.1,138.9,132.0,128.8,127.5,127.4,114.5,109.1$, 48.2 .

\section{4-methoxy-N-(4-methylbenzyl)aniline $(2 \mathrm{~m})^{3}$}<smiles>COc1ccc(NCc2ccc(C)cc2)cc1</smiles>

Light yellow solid, yield $=92 \%, 104 \mathrm{mg}$.

${ }^{1} \mathrm{H}$ NMR $\left(500 \mathrm{MHz}, \mathrm{CDCl}_{3}\right): \delta=7.36(\mathrm{~d}, 2 \mathrm{H}, J=8.0 \mathrm{~Hz}), 7.25(\mathrm{~d}, 2 \mathrm{H}, J=8.0 \mathrm{~Hz}), 6.88(\mathrm{~d}$, $2 \mathrm{H}, J=8.0 \mathrm{~Hz}), 6.69$ (d, 2H, $J=8.5 \mathrm{~Hz}), 4.32$ (s, 2H), 3.83 (s, 3H), 3.71 (brs, 1H), 2.45 (s, $3 \mathrm{H})$.

${ }^{13} \mathrm{C}$ NMR $\left(125 \mathrm{MHz}, \mathrm{CDCl}_{3}\right): \delta=152.1,142.5,136.8,136.6,129.3,127.6,114.9,114.1,55.7$, 48.9, 21.1.

\section{4-bromo-N-(4-methylbenzyl)aniline (2n)}<smiles>Cc1ccc(CNc2ccc(Br)cc2)cc1</smiles>

Light yellow solid, yield $=78 \%, 108 \mathrm{mg}$.

${ }^{1} \mathrm{H}$ NMR $\left(500 \mathrm{MHz}, \mathrm{CDCl}_{3}\right): \delta=7.32-7.28(\mathrm{~m}, 4 \mathrm{H}), 7.24(\mathrm{~d}, 2 \mathrm{H}, J=8.0 \mathrm{~Hz}), 6.55(\mathrm{~d}, 2 \mathrm{H}, J=$ $8.5 \mathrm{~Hz}), 4.31$ (s, 2H), 4.10 (brs, 1H), 2.43 (s, 3H).

${ }^{13} \mathrm{C} \mathrm{NMR}\left(125 \mathrm{MHz}, \mathrm{CDCl}_{3}\right): \delta=147.2,137.1,135.9,132.0,129.5,127.5,114.5,109.1,48.0$, 21.2 .

\section{N-benzyl-2-methylpropan-1-amine (20)}<smiles>CC(C)CNCc1ccccc1</smiles>

Yellow solid, yield $=66 \%, 54 \mathrm{mg}$. 
${ }^{1} \mathrm{H}$ NMR $\left(500 \mathrm{MHz}, \mathrm{CDCl}_{3}\right): \delta=7.35(\mathrm{~d}, 4 \mathrm{H}, J=4.0 \mathrm{~Hz}), 7.30-7.27(\mathrm{~m}, 1 \mathrm{H}), 3.82(\mathrm{~s}, 2 \mathrm{H})$, 2.47 (d, 2H, $J=6.5 \mathrm{~Hz}), 1.92$ (brs, 1H), 1.84-1.79 (m, 1H), 0.95 (s, 3H), 0.94 (s, 3H).

${ }^{13} \mathrm{C} \mathrm{NMR}\left(125 \mathrm{MHz}, \mathrm{CDCl}_{3}\right): \delta=150.6,128.5,128.3,127.1,57.5,54.2,28.4,20.8$.

2-methyl-N-(2-methylbenzyl)propan-1-amine (2p)

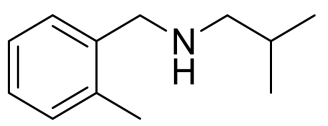

Yellow solid, yield $=79 \%, 70 \mathrm{mg}$.

${ }^{1} \mathrm{H}$ NMR (500 MHz, $\left.\mathrm{CDCl}_{3}\right): \delta=7.36-7.34(\mathrm{~m}, 1 \mathrm{H}), 7.22-7.20(\mathrm{~m}, 3 \mathrm{H}), 3.80(\mathrm{~s}, 2 \mathrm{H}), 2.53(\mathrm{~d}$, $2 \mathrm{H}, J=7.0 \mathrm{~Hz}), 2.39$ (s, 3H), 1.88-1.80 (m, 1H), 1.65 (brs, 1H), 0.98 (s, 3H), 0.97 (s, 3H).

${ }^{13} \mathrm{C} \mathrm{NMR}\left(125 \mathrm{MHz}, \mathrm{CDCl}_{3}\right): \delta=138.6,136.4,130.4,128.4,127.0,126.0,58.0,51.9,28.5$, 20.9, 19.1.

\section{4-methyl-N-(4-nitrobenzyl)aniline (2q) ${ }^{7}$}<smiles>Cc1ccc(NCc2ccc([N+](=O)[O-])cc2)cc1</smiles>

Yellow solid, yield $=51 \%, 62 \mathrm{mg}$.

${ }^{1} \mathrm{H}$ NMR $\left(500 \mathrm{MHz}, \mathrm{CDCl}_{3}\right): \delta=8.20(\mathrm{~d}, 2 \mathrm{H}, J=8.0 \mathrm{~Hz}), 7.56(\mathrm{~d}, 2 \mathrm{H}, J=8.5 \mathrm{~Hz}), 7.02(\mathrm{~d}$, $2 \mathrm{H}, J=8.0 \mathrm{~Hz}), 6.54(\mathrm{~d}, 2 \mathrm{H}, J=8.5 \mathrm{~Hz}), 4.48(\mathrm{~s}, 2 \mathrm{H}), 2.27(\mathrm{~s}, 3 \mathrm{H})$.

${ }^{13} \mathrm{C} \mathrm{NMR}\left(125 \mathrm{MHz}, \mathrm{CDCl}_{3}\right): \delta=147.9,147.2,145.1,130.0,127.8,127.5,123.9,113.2,48.0$, 20.5 .

\section{4-((phenylamino)methyl)benzonitrile $(2 \mathrm{r})^{8}$}<smiles>N#Cc1ccc(CNc2ccccc2)cc1</smiles>

Yellow solid, yield $=67 \%, 70 \mathrm{mg}$.

${ }^{1} \mathrm{H}$ NMR (500 MHz, $\left.\mathrm{CDCl}_{3}\right): \delta=7.64-7.63(\mathrm{~m}, 2 \mathrm{H}), 7.51(\mathrm{~d}, 2 \mathrm{H}, J=8.0 \mathrm{~Hz}), 7.22(\mathrm{t}, 2 \mathrm{H}, J=$ $8.5 \mathrm{~Hz}), 6.79(\mathrm{t}, 1 \mathrm{H}, J=7.0 \mathrm{~Hz}), 6.62(\mathrm{~d}, 2 \mathrm{H}, J=7.5 \mathrm{~Hz}), 4.46$ (s, 2H), 4.15 (brs, 1H).

${ }^{13} \mathrm{C}$ NMR $\left(125 \mathrm{MHz}, \mathrm{CDCl}_{3}\right): \delta=147.4,145.5,132.4,129.4,127.7,119.0,118.1,112.9$, 110.8, 47.7.

methyl 4-((p-tolylamino)methyl)benzoate (2s) ${ }^{7}$<smiles>CC(=O)c1ccc(CNc2ccc(C)cc2)cc1</smiles>

Light yellow solid, yield $=67 \%, 85 \mathrm{mg}$. 
${ }^{1} \mathrm{H}$ NMR $\left(500 \mathrm{MHz}, \mathrm{CDCl}_{3}\right): \delta=8.06(\mathrm{~d}, 2 \mathrm{H}, J=7.5 \mathrm{~Hz}), 7.47(\mathrm{~d}, 2 \mathrm{H}, J=7.5 \mathrm{~Hz}), 7.03(\mathrm{~d}$, $2 \mathrm{H}, J=8.0 \mathrm{~Hz}), 6.59$ (d, 2H, $J=7.5 \mathrm{~Hz}), 4.41$ (s, 2H), 4.10 (brs, 1H), 3.96 (s, 3H), 2.30 (s, $3 \mathrm{H})$.

${ }^{13} \mathrm{C}$ NMR (125 MHz, $\left.\mathrm{CDCl}_{3}\right): \delta=167.0,145.5,145.3,129.9,129.8,129.0,127.2,127.0$, $113.1,52.1,48.3,20.4$.

methyl 2-(benzhydrylamino)acetate (2t)<smiles>COC(=O)CNC(c1ccccc1)c1ccccc1</smiles>

Yellow oil, yield $=65 \%, 83 \mathrm{mg}$.

${ }^{1} \mathrm{H}$ NMR $\left(500 \mathrm{MHz}, \mathrm{CDCl}_{3}\right): \delta=8.49-8.47(\mathrm{~m}, 4 \mathrm{H}), 7.36(\mathrm{t}, 4 \mathrm{H}, J=7.5 \mathrm{~Hz}), 7.29-7.26(\mathrm{~m}$, $2 \mathrm{H}), 4.95(\mathrm{~s}, 1 \mathrm{H}), 3.76(\mathrm{~s}, 3 \mathrm{H}), 3.45(\mathrm{~s}, 2 \mathrm{H}), 2.26$ (brs, $1 \mathrm{H})$.

${ }^{13} \mathrm{C} \mathrm{NMR}\left(125 \mathrm{MHz}, \mathrm{CDCl}_{3}\right): \delta=173.1,143.3,128.7,127.5,127.3,66.6,51.9,48.9$.

(E)-N-cinnamylaniline (2u) ${ }^{9}$<smiles>C(=C/c1ccccc1)\CNc1ccccc1</smiles>

Light yellow oil, yield $=75 \%, 78 \mathrm{mg}$.

${ }^{1} \mathrm{H}$ NMR $\left(500 \mathrm{MHz}, \mathrm{CDCl}_{3}\right): \delta=7.49-7.28(\mathrm{~m}, 7 \mathrm{H}), 6.86-6.70(\mathrm{~m}, 4 \mathrm{H}), 6.45-6.40(\mathrm{~m}, 1 \mathrm{H})$, 4.02-4.01 (m, 2H), 3.79 (brs, $1 \mathrm{H})$.

${ }^{13} \mathrm{C}$ NMR $\left(125 \mathrm{MHz}, \mathrm{CDCl}_{3}\right): \delta=148.1,137.0,131.6,129.4,128.7,127.6,127.1,126.4$, $117.7,113.2,46.3$.

\section{N-((5-methylthiophen-2-yl)methyl)aniline (2v)}<smiles>Cc1ccc(CNc2ccccc2)s1</smiles>

Light yellow oil, yield $=90 \%, 91 \mathrm{mg}$.

${ }^{1} \mathrm{H}$ NMR $\left(500 \mathrm{MHz}, \mathrm{CDCl}_{3}\right): \delta=7.31-7.28(\mathrm{~m}, 2 \mathrm{H}), 6.88-6.84(\mathrm{~m}, 2 \mathrm{H}), 6.76(\mathrm{~d}, 2 \mathrm{H}, J=7.5$ $\mathrm{Hz}), 6.70-6.69(\mathrm{~m}, 1 \mathrm{H}), 4.50(\mathrm{~s}, 2 \mathrm{H}), 2.54(\mathrm{~s}, 3 \mathrm{H})$.

${ }^{13} \mathrm{C}$ NMR (125 MHz, $\left.\mathrm{CDCl}_{3}\right): \delta=147.7,140.4,139.2,129.3,125.1,124.9,118.1,113.2,43.7$, 15.5. 
<smiles>c1ccc(NCc2ccc3ccccc3c2)cc1</smiles>

Light yellow solid, yield $=71 \%, 83 \mathrm{mg}$.

${ }^{1} \mathrm{H}$ NMR (500 MHz, $\left.\mathrm{CDCl}_{3}\right): \delta=7.93-7.89(\mathrm{~m}, 4 \mathrm{H}), 7.58-7.55(\mathrm{~m}, 3 \mathrm{H}), 7.30-7.27(\mathrm{~m}, 2 \mathrm{H})$, 6.86-6.75 (m, 3H), 4.55 (s, 2H), 4.15 (brs, $1 \mathrm{H})$.

${ }^{13} \mathrm{C}$ NMR $\left(125 \mathrm{MHz}, \mathrm{CDCl}_{3}\right): \delta=148.2,137.0,133.6,132.9,129.4,128.5,127.9,127.8$, $126.3,126.1,125.9,117.8,113.1,48.6$.

\section{1-(benzo[d][1,3]dioxol-5-yl)-N-benzylmethanamine $(2 x)^{10}$}<smiles>c1ccc(CNCc2ccc3c(c2)OCO3)cc1</smiles>

White solid, yield $=77 \%, 93 \mathrm{mg}$.

${ }^{1} \mathrm{H}$ NMR (500 MHz, $\left.\mathrm{CDCl}_{3}\right): \delta=7.37-7.29(\mathrm{~m}, 5 \mathrm{H}), 6.90$ (s, 1H), 6.82-6.78 (m, 2H), $5.96(\mathrm{~s}$, $2 \mathrm{H}), 3.82(\mathrm{~s}, 2 \mathrm{H}), 3.74(\mathrm{~s}, 2 \mathrm{H})$.

${ }^{13} \mathrm{C}$ NMR $\left(125 \mathrm{MHz}, \mathrm{CDCl}_{3}\right): \delta=147.9,146.8,139.9,133.8,128.6,128.4,127.3,121.6$, $108.9,108.3,101.1,52.9,52.8$.

\section{$N$-[(Ferrocenyl)methyl]aniline $(2 \mathrm{y})^{8}$}

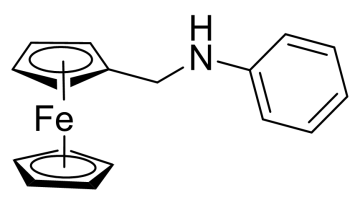

Brown solid, yield $=60 \%, 96 \mathrm{mg}$.

${ }^{1} \mathrm{H}$ NMR $\left(500 \mathrm{MHz}, \mathrm{CDCl}_{3}\right): \delta=7.28(\mathrm{t}, 2 \mathrm{H}, J=7.5 \mathrm{~Hz}), 6.82-6.73(\mathrm{~m}, 3 \mathrm{H}), 4.32-4.17(\mathrm{~m}$, $9 \mathrm{H}), 4.02$ (s, 2H), 3.92 (brs, 1H).

${ }^{13} \mathrm{C}$ NMR $\left(125 \mathrm{MHz}, \mathrm{CDCl}_{3}\right): \delta=148.4,129.4,117.6,112.9,86.5,68.6,68.2,68.0,43.5$.

\section{3-(4-(benzylamino)phenyl)-3-ethylpiperidine-2,6-dione (2z)}<smiles>CCC1(c2ccc(NCc3ccccc3)cc2)CCC(=O)NC1=O</smiles>

Colorless oil, In DMC: yield $=72 \%, 116 \mathrm{mg}$.

${ }^{1} \mathrm{H}$ NMR $\left(500 \mathrm{MHz}, \mathrm{CDCl}_{3}\right): \delta=8.5(\mathrm{~s}, 1 \mathrm{H}), 7.39-7.29(\mathrm{~m}, 5 \mathrm{H}), 7.09(\mathrm{~d}, 2 \mathrm{H}, J=8.5 \mathrm{~Hz})$, $6.64(\mathrm{~d}, 2 \mathrm{H}, J=8.5 \mathrm{~Hz}), 4.33(\mathrm{~s}, 2 \mathrm{H}), 2.60-2.57(\mathrm{~m}, 1 \mathrm{H}), 2.50-2.44(\mathrm{~m}, 1 \mathrm{H}), 2.33-2.29$ (m, $1 \mathrm{H}), 2.20-2.13(\mathrm{~m}, 1 \mathrm{H}), 2.04-2.00(\mathrm{~m}, 1 \mathrm{H}), 1.92-1.87(\mathrm{~m}, 1 \mathrm{H}), 0.87(\mathrm{t}, 3 \mathrm{H}, J=6.5 \mathrm{~Hz})$.

${ }^{13} \mathrm{C}$ NMR $\left(125 \mathrm{MHz}, \mathrm{CDCl}_{3}\right): \delta=176.0,173.2,147.3,139.1,128.8,127.7,127.5,127.3$, $127.2,113.3,50.3,48.4,33.1,29.5,26.9,9.1$. 
HRMS (EI): $m / z$ calcd for $\mathrm{C}_{22} \mathrm{H}_{23} \mathrm{~N}_{2} \mathrm{O}_{2}[\mathrm{M}+\mathrm{H}]^{+} 323.1760$, found 323.1743 . 


\section{References}

1. Carden, J. L.; Gierlichs, L. J.; Wass, D. F.; Browne, D. L.; Melen, R. L. Unlocking the catalytic potential of tris $(3,4,5$-trifluorophenyl) borane with microwave irradiation. Chem. Commun., 2019, 55, 318-321.

2. Wang, R.; Han, X.; Xu, J.; Liu, P.; Li, F. Transfer hydrogenation of ketones and imines with methanol under base-free conditions catalyzed by an anionic metal-ligand bifunctional iridium catalyst. J. Org. Chem., 2020, 85, 2242-2249.

3. Mamidala, R.; Mukundam, V.; Dhanunjayarao, K.; Venkatasubbaiah, K. Cyclometalated palladium pre-catalyst for $\mathrm{N}$-alkylation of amines using alcohols and regioselective alkylation of sulfanilamide using aryl alcohols. Tetrahedron, 2017, 73, 2225-2233.

4. Kumar, A.; Sharma, S.; Maurya, Ram A. M. Single nucleotide-catalyzed biomimetic reductive amination. Adv. Synth. Catal., 2010, 352, 2227-2232.

5. Gour, J.; Gatadi, S.; Malasala, S.; Yaddanpudi, M. V.; Nanduri, S. A Microwave-assisted $\mathrm{SmI}_{2}$-catalyzed direct N-alkylation of anilines with alcohols. J. Org. Chem., 2019, 84, $7488-7494$.

6. Laroche, B.; Ishitani, H.; Kobayashi, S. Direct Reductive Amination of carbonyl compounds with $\mathrm{H}_{2}$ using heterogeneous catalysts in continuous flow as an alternative to N-alkylation with alkyl halides. Adv. Synth. Catal., 2018, 360, 4699-4704.

7. Li, B.; Sortais, J.-B.; Darcel, C.; Dixneuf, P. H. Amine synthesis through mild catalytic hydrosilylation of imines using polymethylhydroxysiloxane and $\left[\mathrm{RuCl}_{2}(\text { arene) }]_{2}\right.$ catalysts. ChemSusChem, 2012, 5, 396-399.

8. Sousa, S. C. A.; Fernandes, A. C. Efficient and highly chemoselective direct reductive amination of aldehydes using the system silane/oxorhenium complexes. Adv. Synth. Catal., 2010, 352, 2218-2226.

9. Maya, R. J.; Poulose, S.; John, J.; Luxmi V. Direct reductive amination of aldehydes via environmentally benign bentonite-gold nanohybrid catalysis. Adv. Synth Catal., 2017, 359, 1177-1184.

10. Cheng, C.; Brookhart, M. Iridium-catalyzed reduction of secondary amides to secondary amines and imines by diethylsilane. J. Am. Chem. Soc. 2012, 134, 11304-11307. 


\section{$\underline{1 H \text { and } 13 C \text { NMR Spectra }}$}

N-benzylaniline (2a)<smiles>c1ccc(CNc2ccccc2)cc1</smiles>
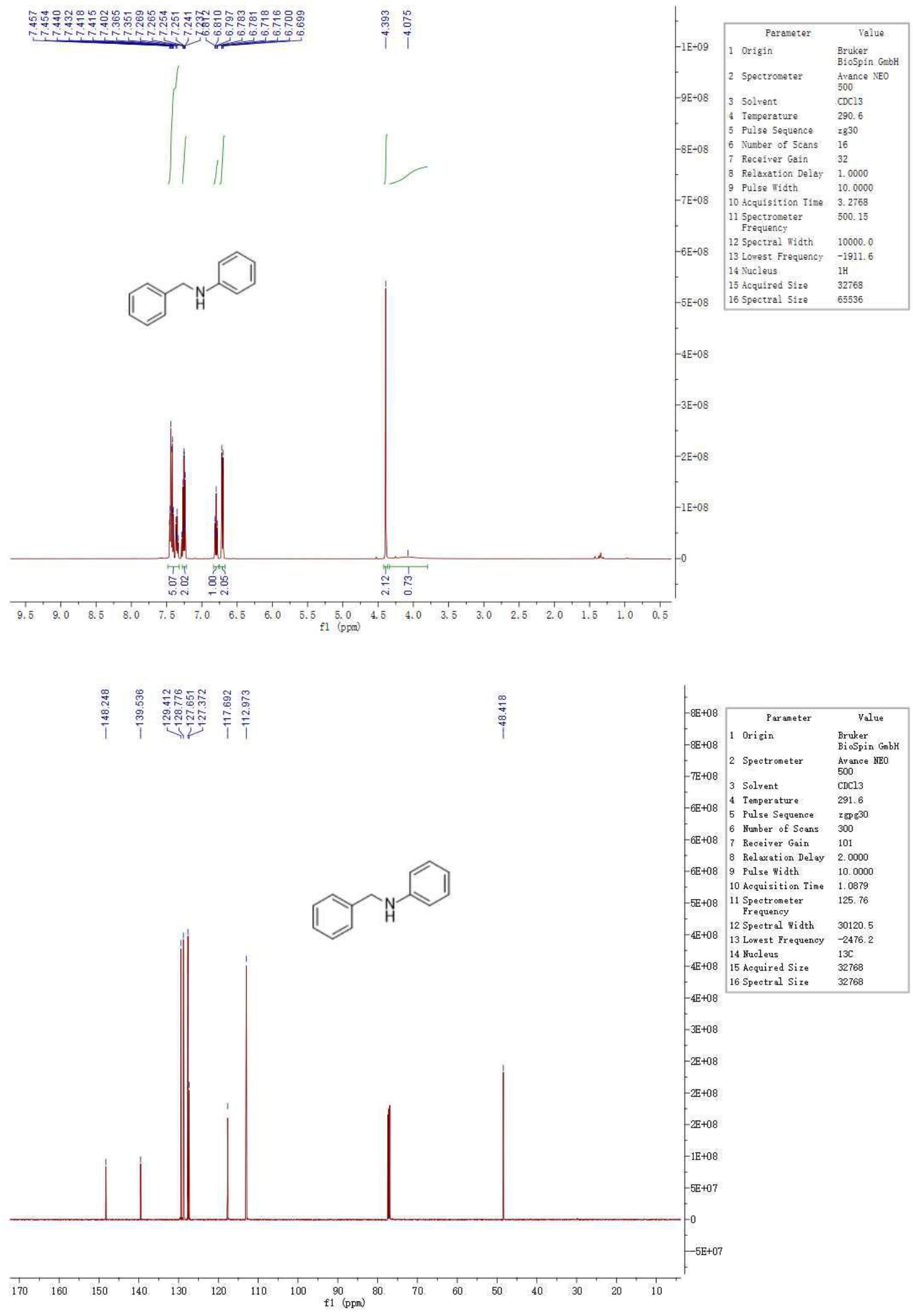


\section{N-(4-methylbenzyl)aniline (2b)}
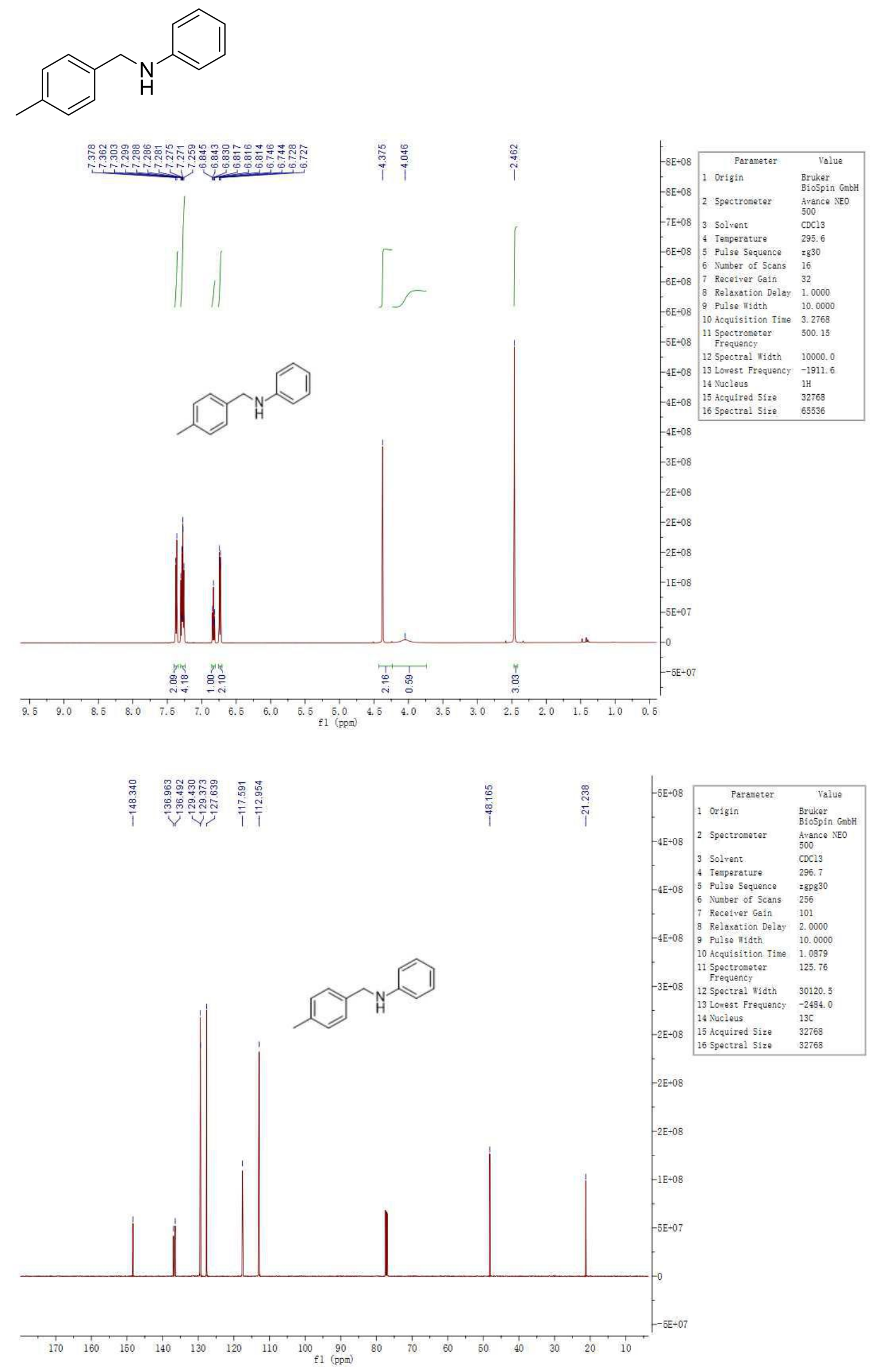


\section{N-(3-methylbenzyl)aniline (2c)}<smiles>Cc1cccc(CNc2ccccc2)c1</smiles>
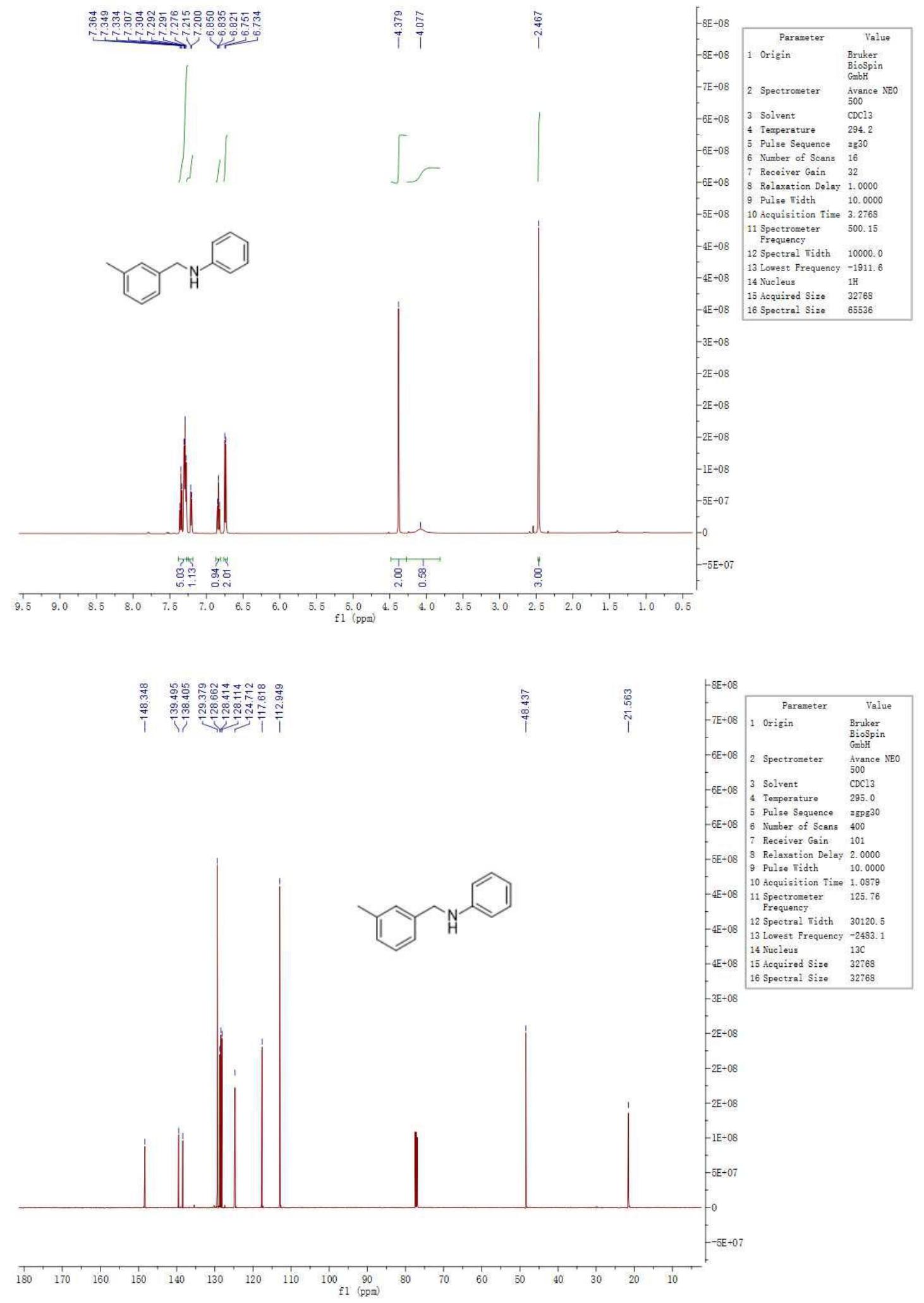
N-(2-methylbenzyl)aniline (2d)<smiles>Cc1ccccc1CNc1ccccc1</smiles>
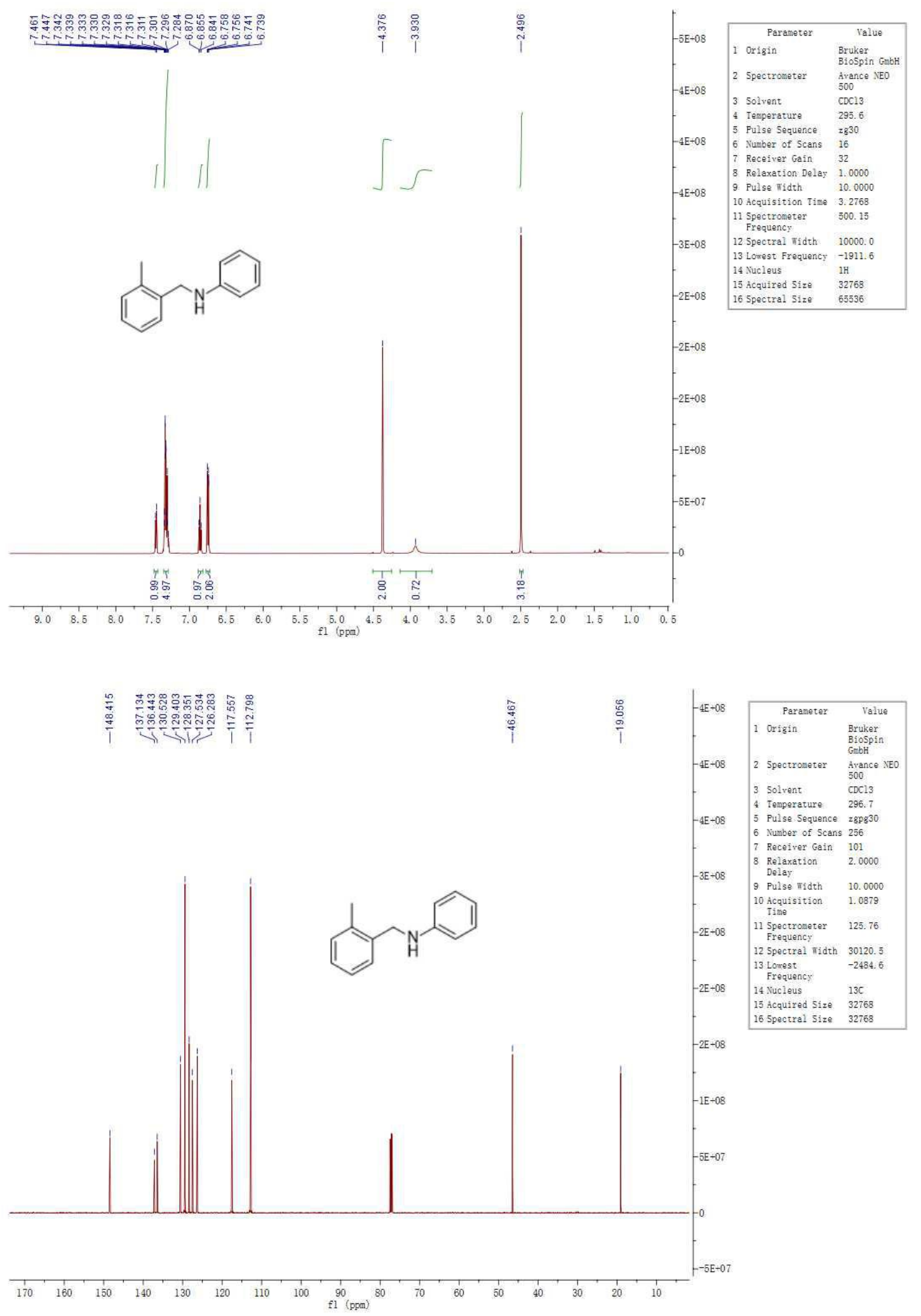


\section{N-(4-methoxybenzyl)aniline (2e)}<smiles>COc1ccc(CNc2ccccc2)cc1</smiles>

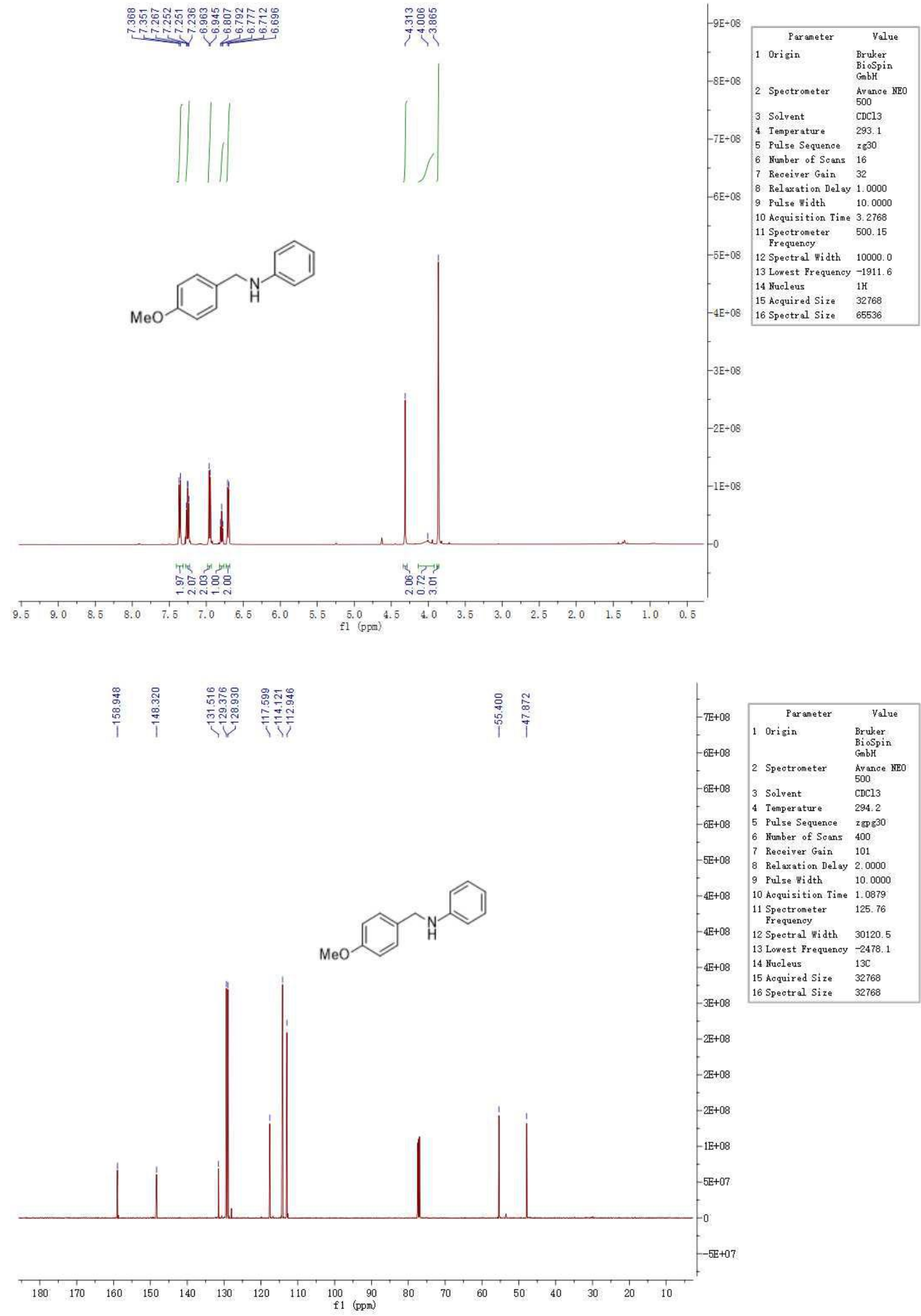




\section{N-(3-methoxybenzyl)aniline (2f)}<smiles>COc1cccc(CNc2ccccc2)c1</smiles>

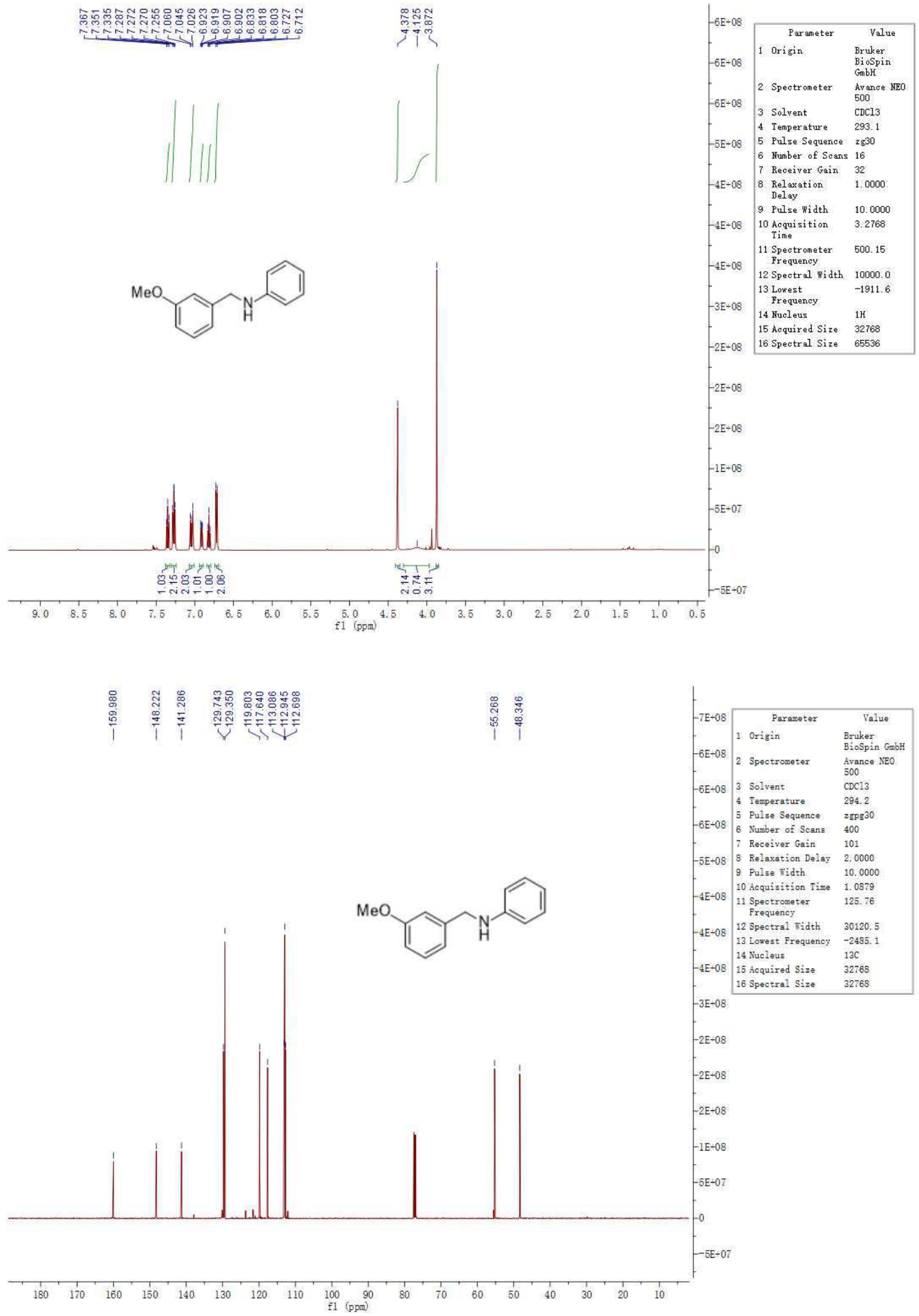




\section{N-(2-methoxybenzyl)aniline (2g)}<smiles>COc1ccccc1CNc1ccccc1</smiles>
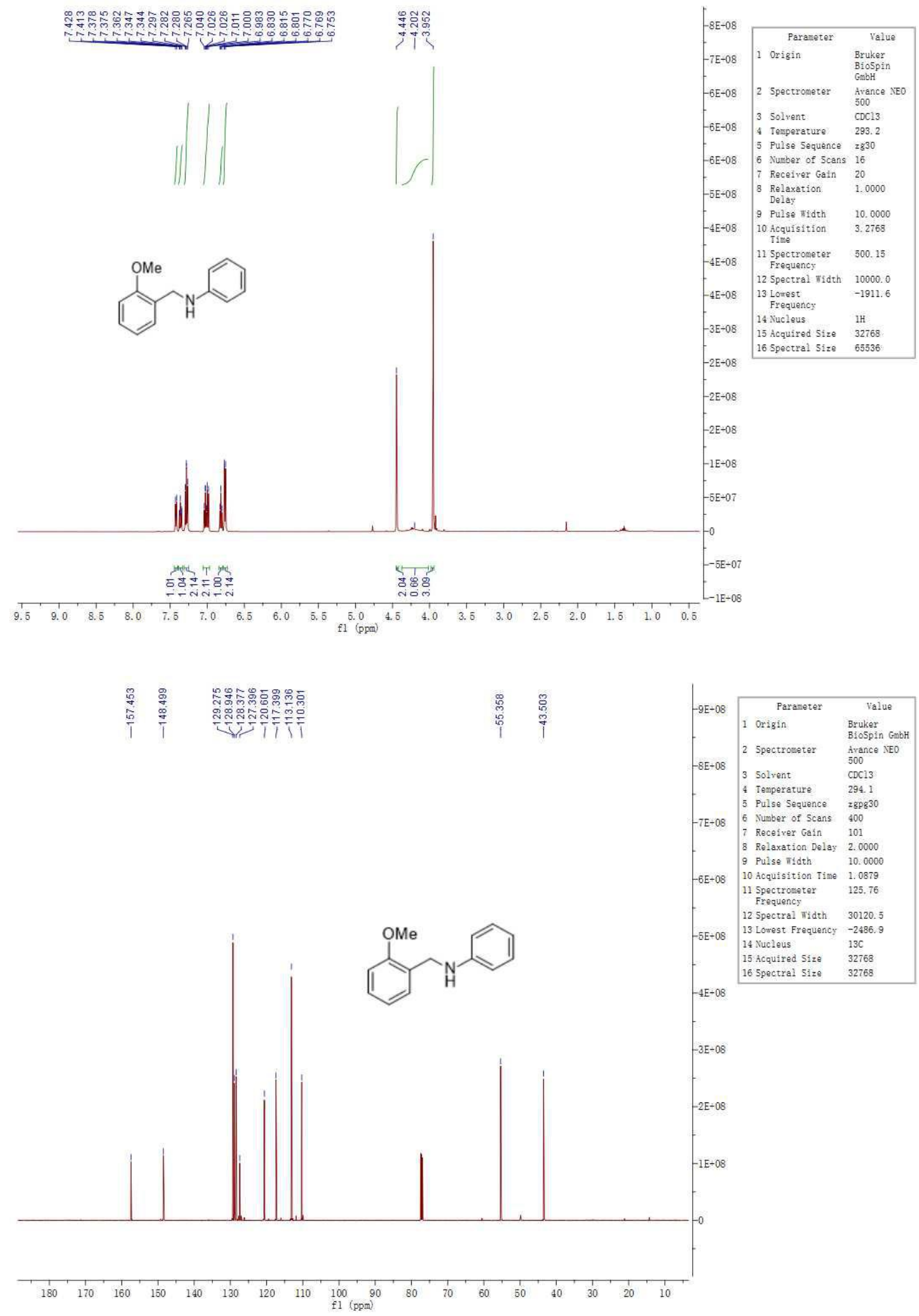


\section{N-(4-bromobenzyl)aniline (2h)}<smiles>Brc1ccc(CNc2ccccc2)cc1</smiles>

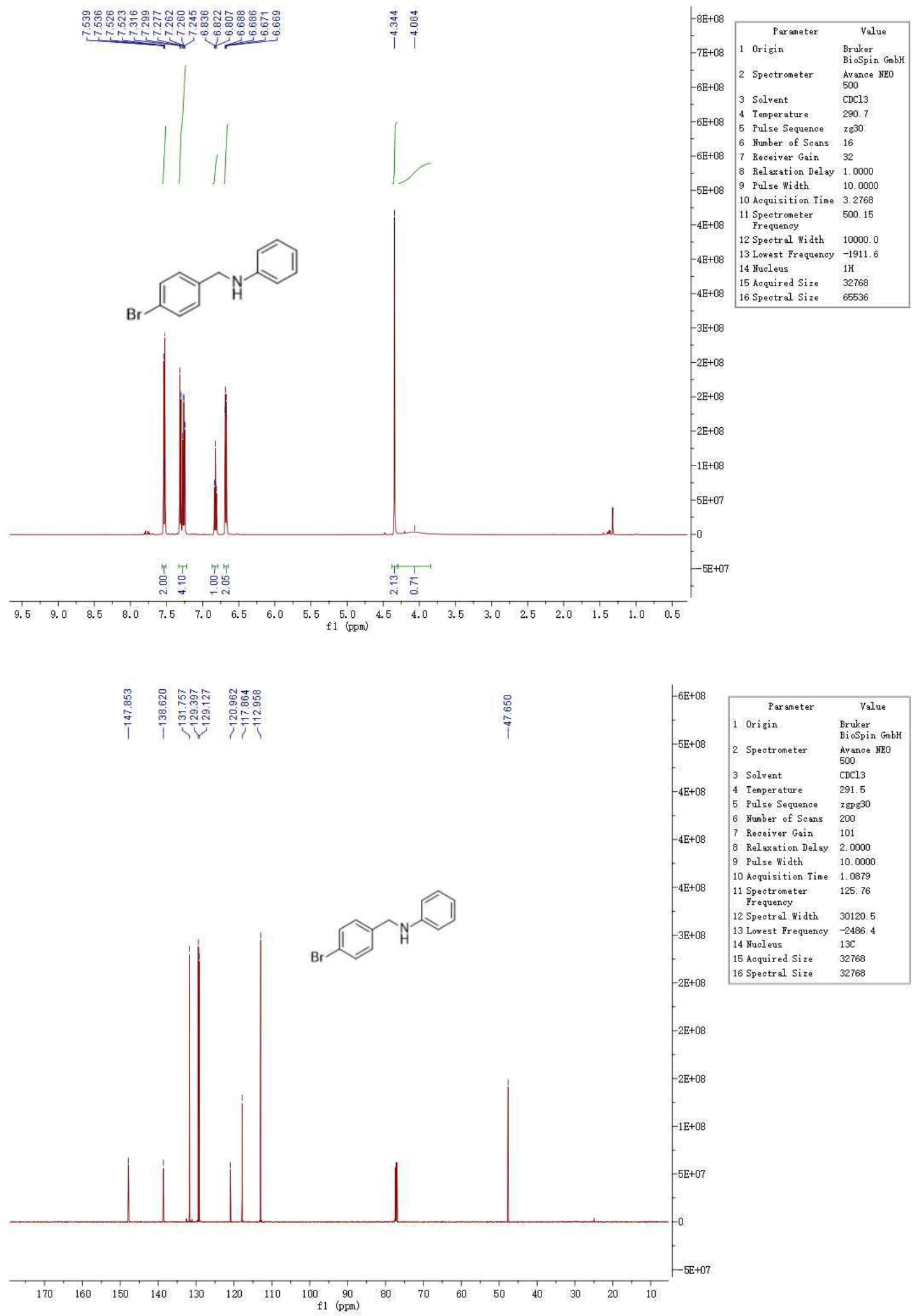




\section{N-(4-(trifluoromethyl)benzyl)aniline (2i)}<smiles>FC(F)(F)c1ccc(CNc2ccccc2)cc1</smiles>
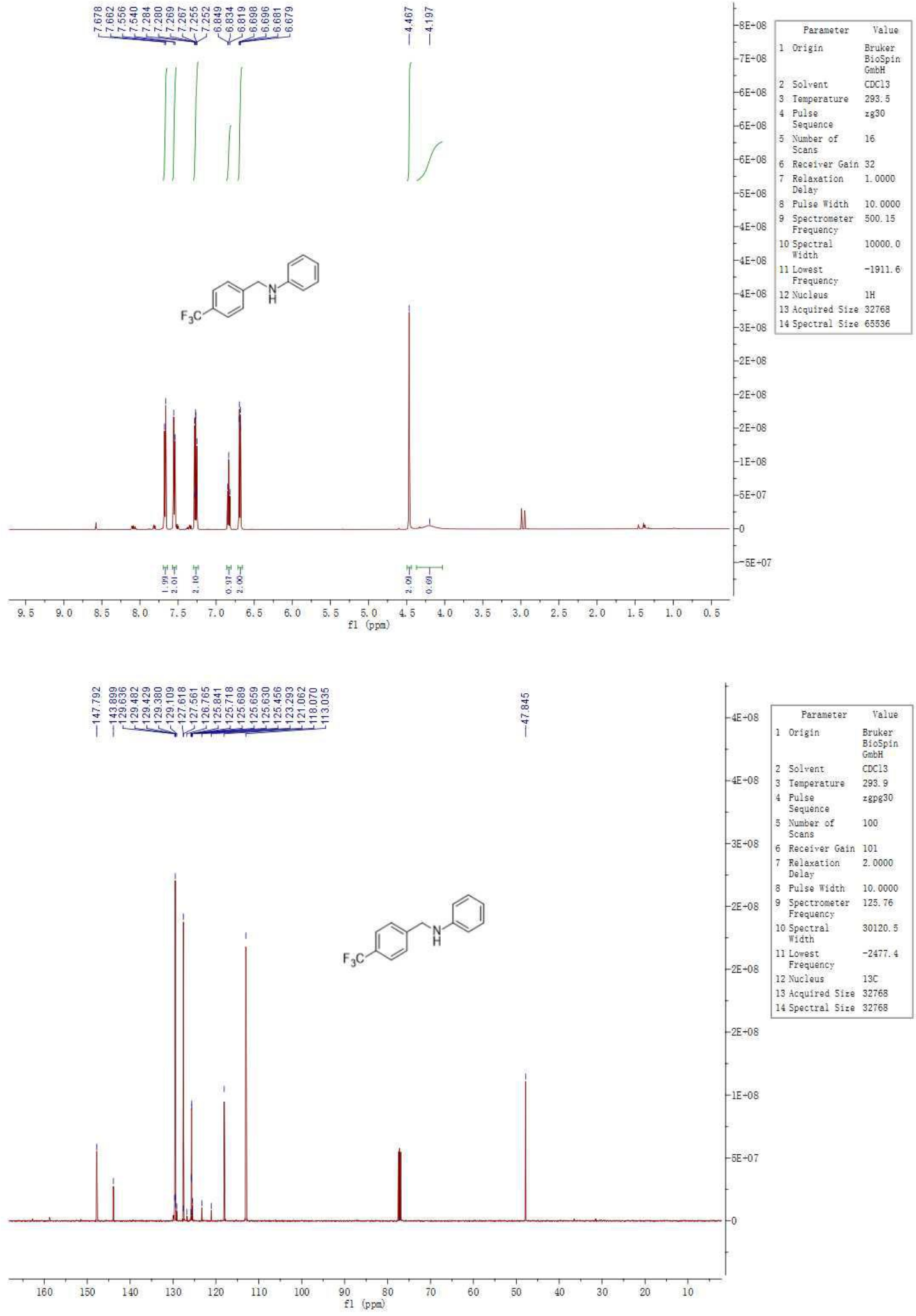
N-benzyl-4-methylaniline (2j)<smiles>Cc1ccc(NCc2ccccc2)cc1</smiles>
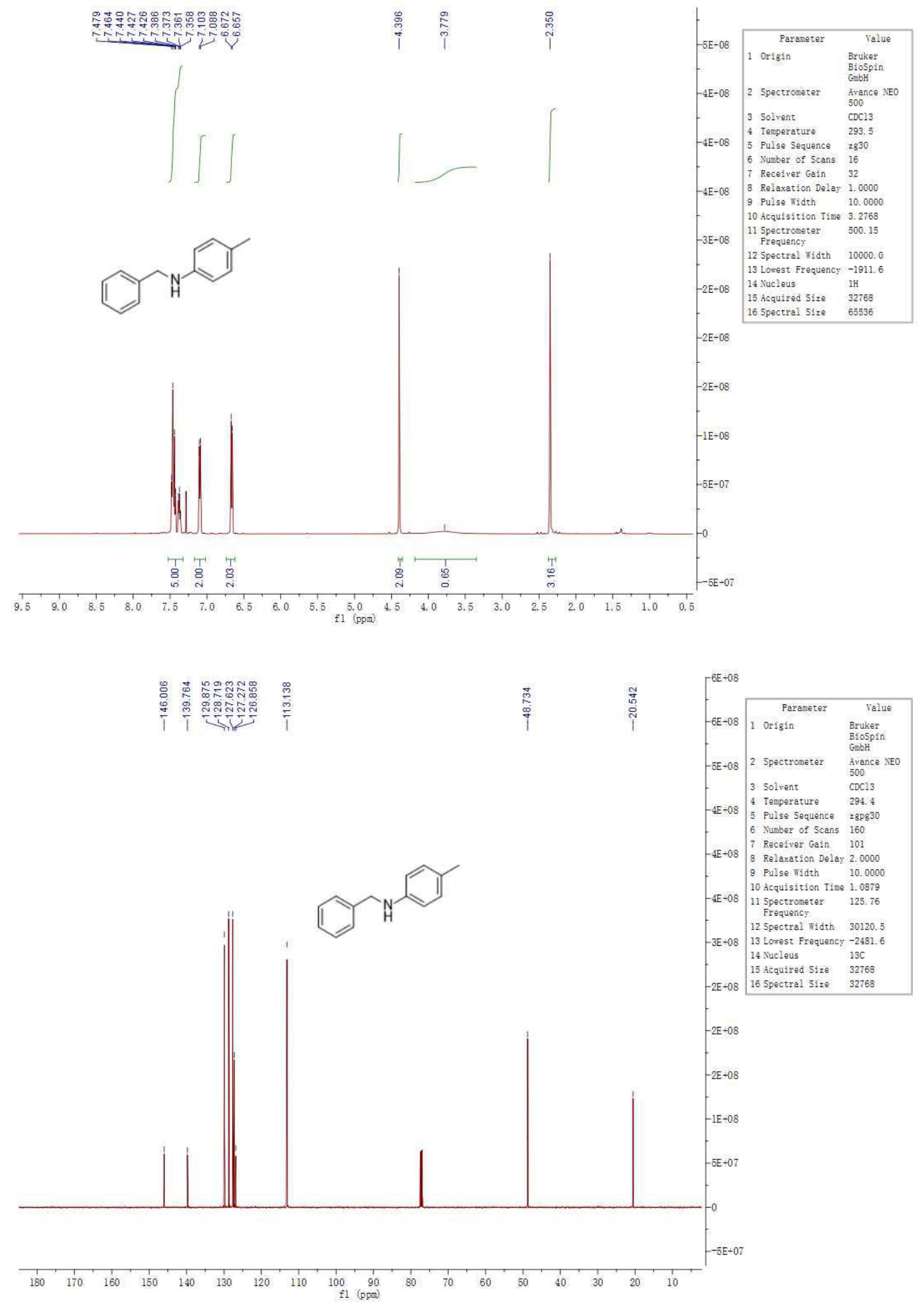


\section{N-benzyl-4-methoxyaniline (2k)}<smiles>COc1ccc(NCc2ccccc2)cc1</smiles>

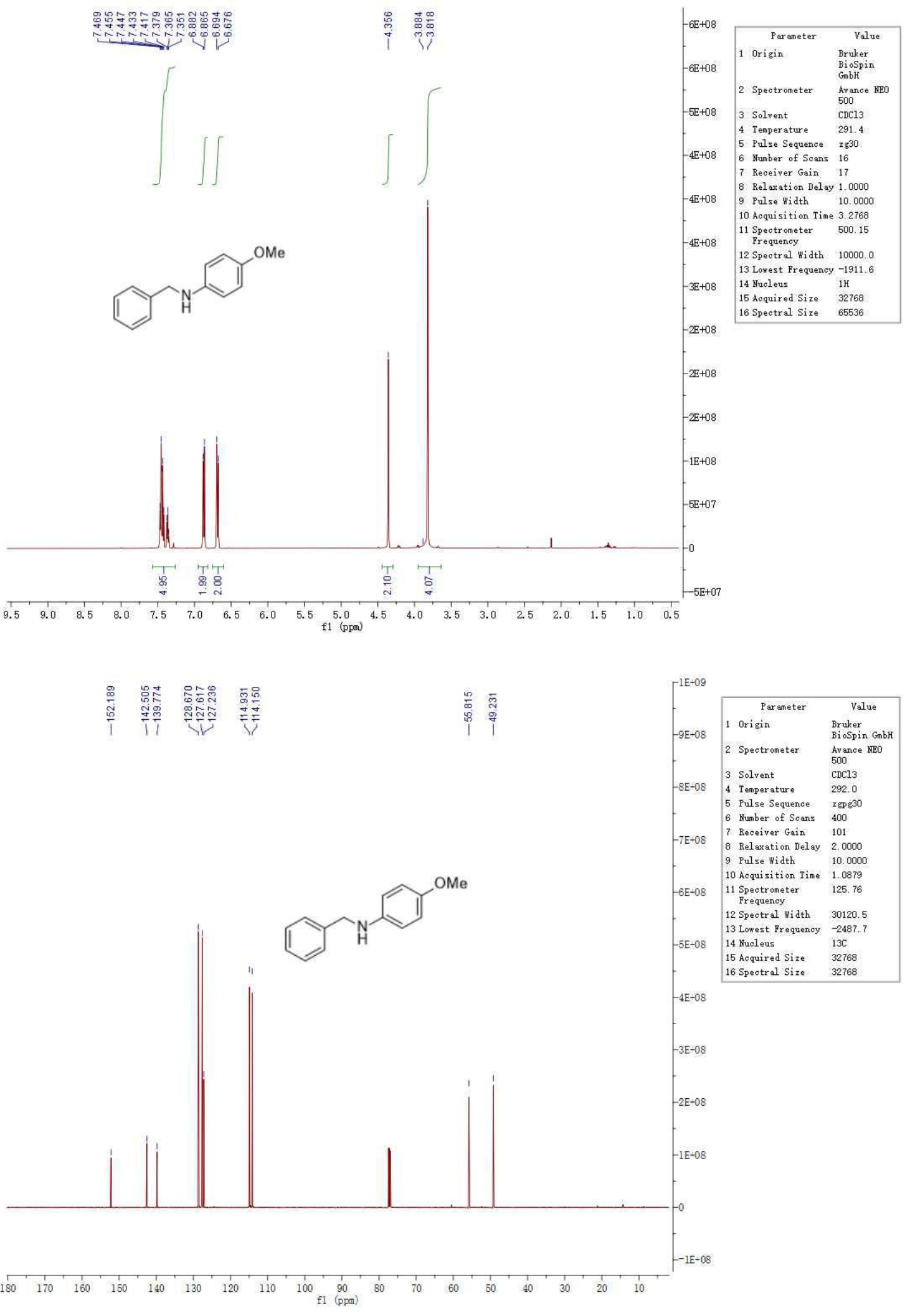




\section{N-benzyl-4-bromoaniline (2I)}<smiles>Brc1ccc(NCc2ccccc2)cc1</smiles>
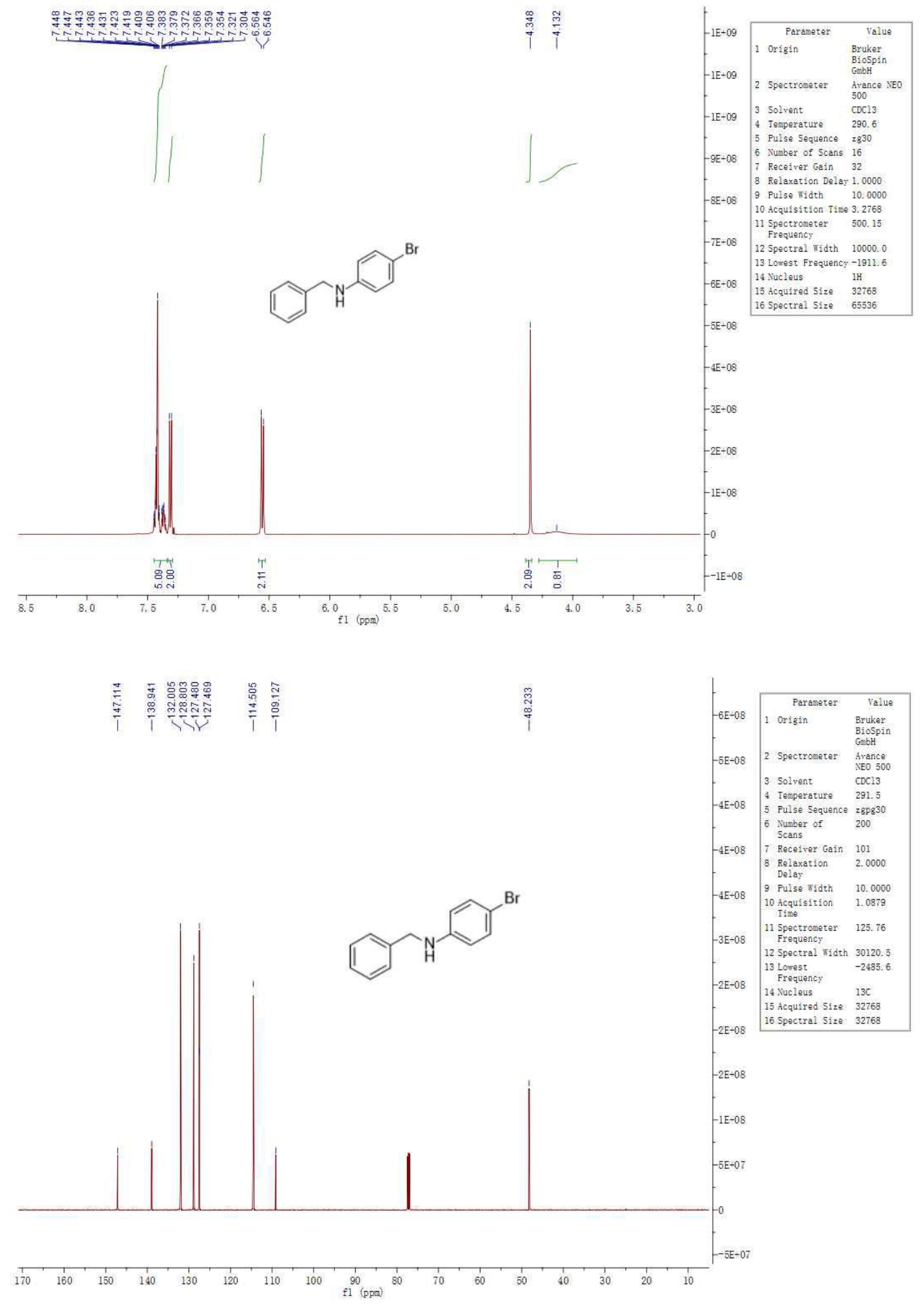


\section{4-methoxy-N-(4-methylbenzyl)aniline (2m)}<smiles>COc1ccc(NCc2ccc(C)cc2)cc1</smiles>

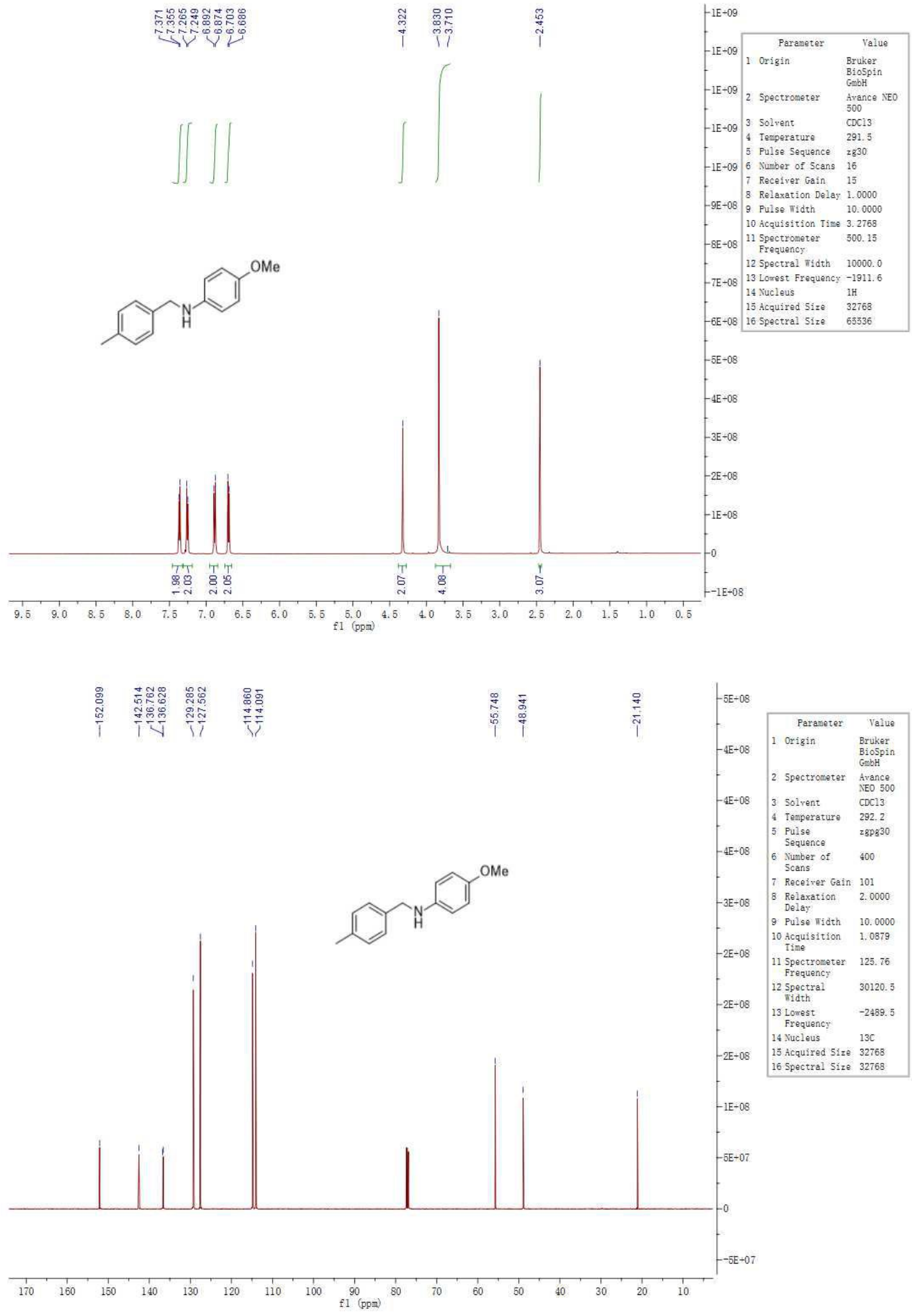


4-bromo-N-(4-methylbenzyl)aniline (2n)<smiles>Cc1ccc(CNc2ccc(Br)cc2)cc1</smiles>

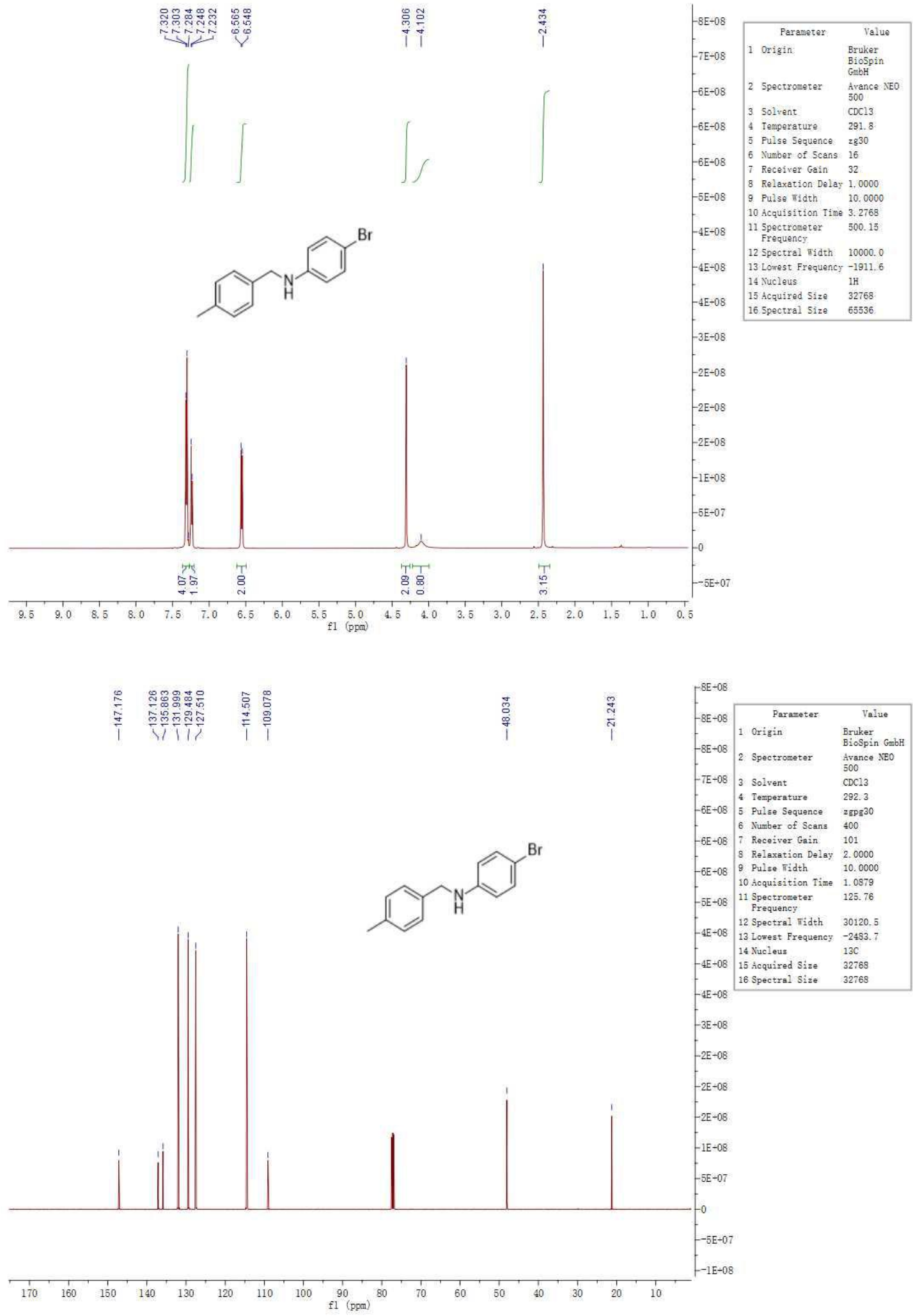


N-benzyl-2-methylpropan-1-amine (2o)

$\curvearrowright \mathrm{N}$

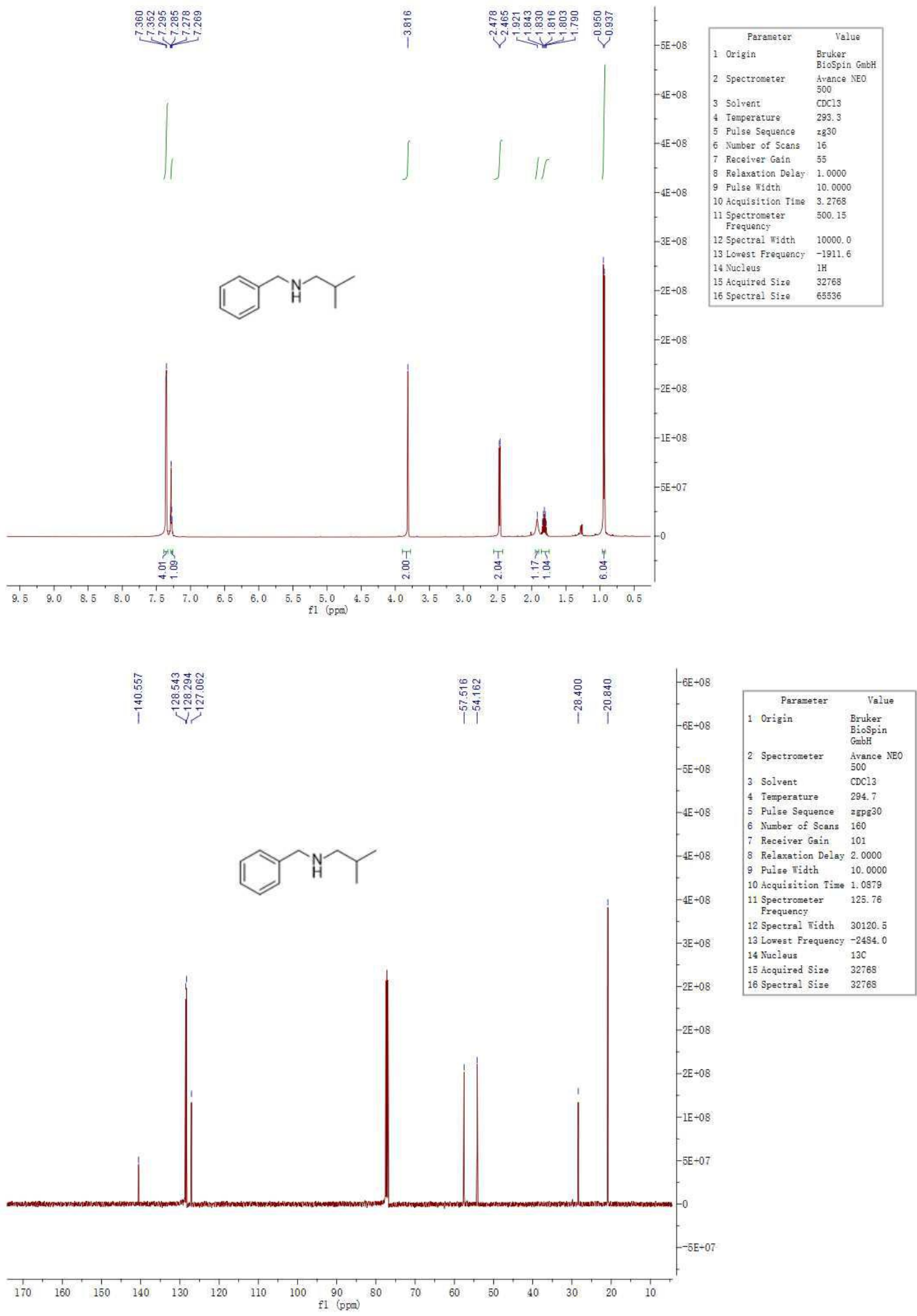


2-methyl-N-(2-methylbenzyl)propan-1-amine (2p)<smiles>Cc1ccccc1CNCC(C)C</smiles>
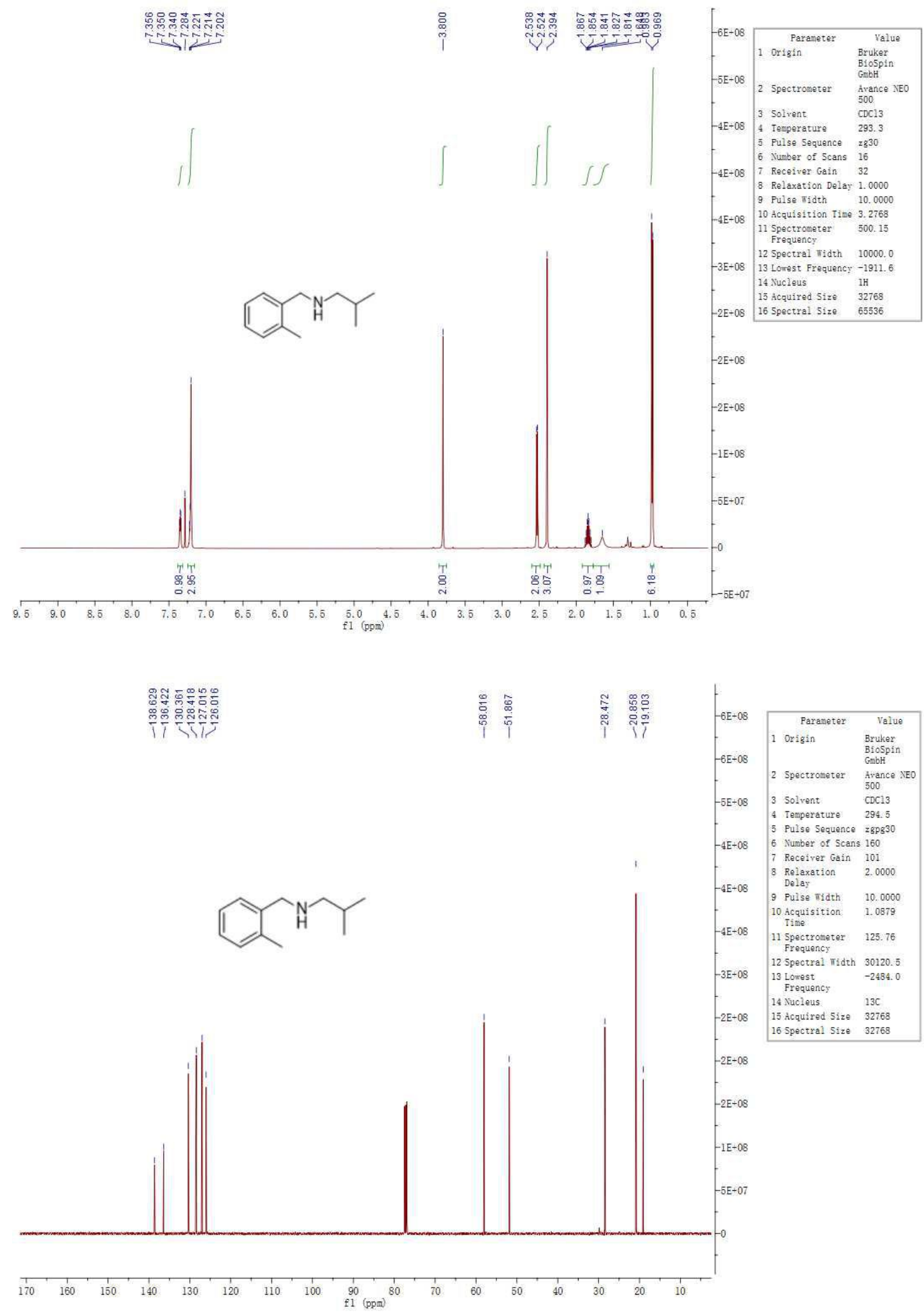


\section{4-methyl-N-(4-nitrobenzyl)aniline (2q)}<smiles>Cc1ccc(NCc2ccc([N+](=O)[O-])cc2)cc1</smiles>
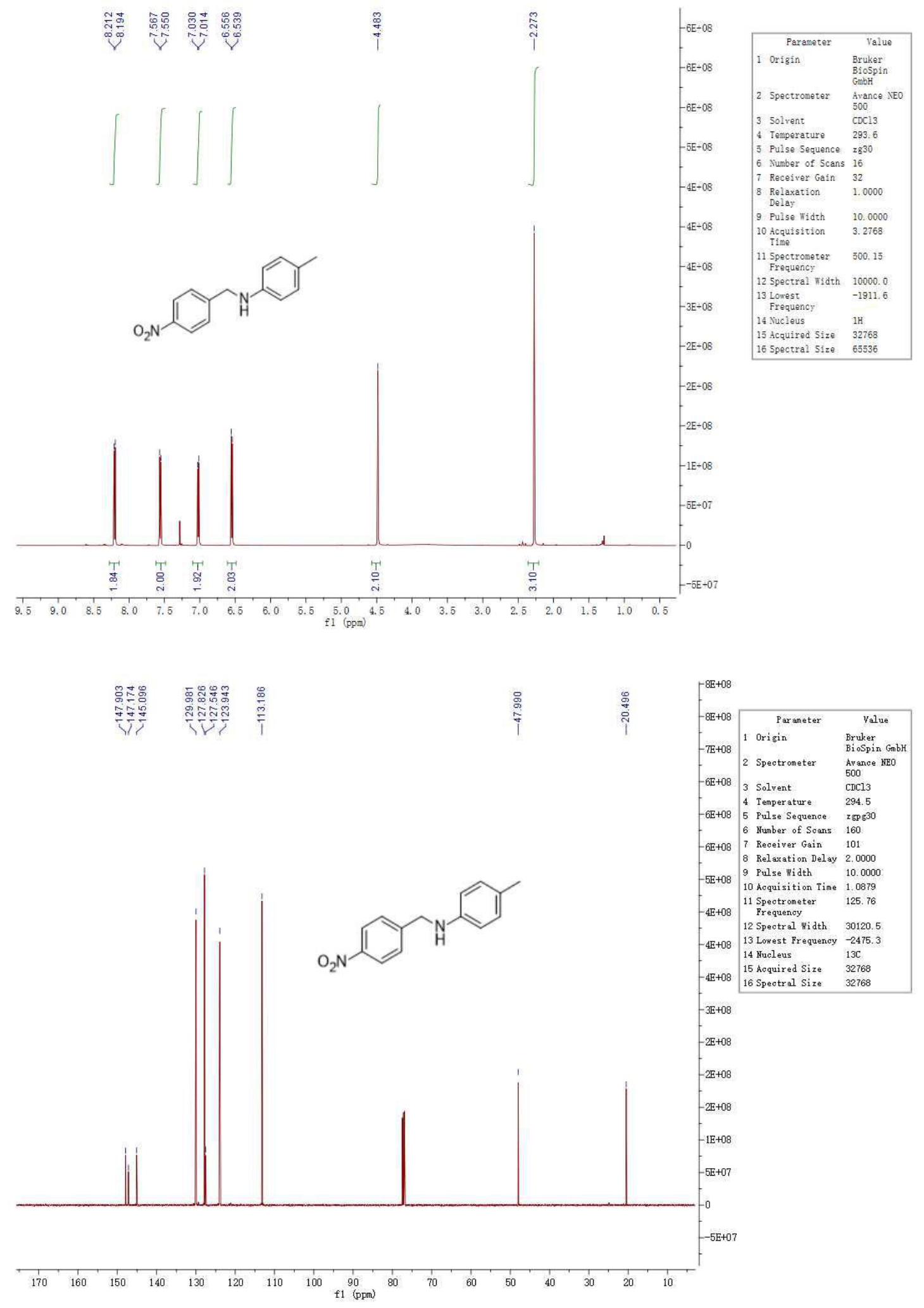
4-((phenylamino)methyl)benzonitrile (2r)<smiles>N#Cc1ccc(CNc2ccccc2)cc1</smiles>
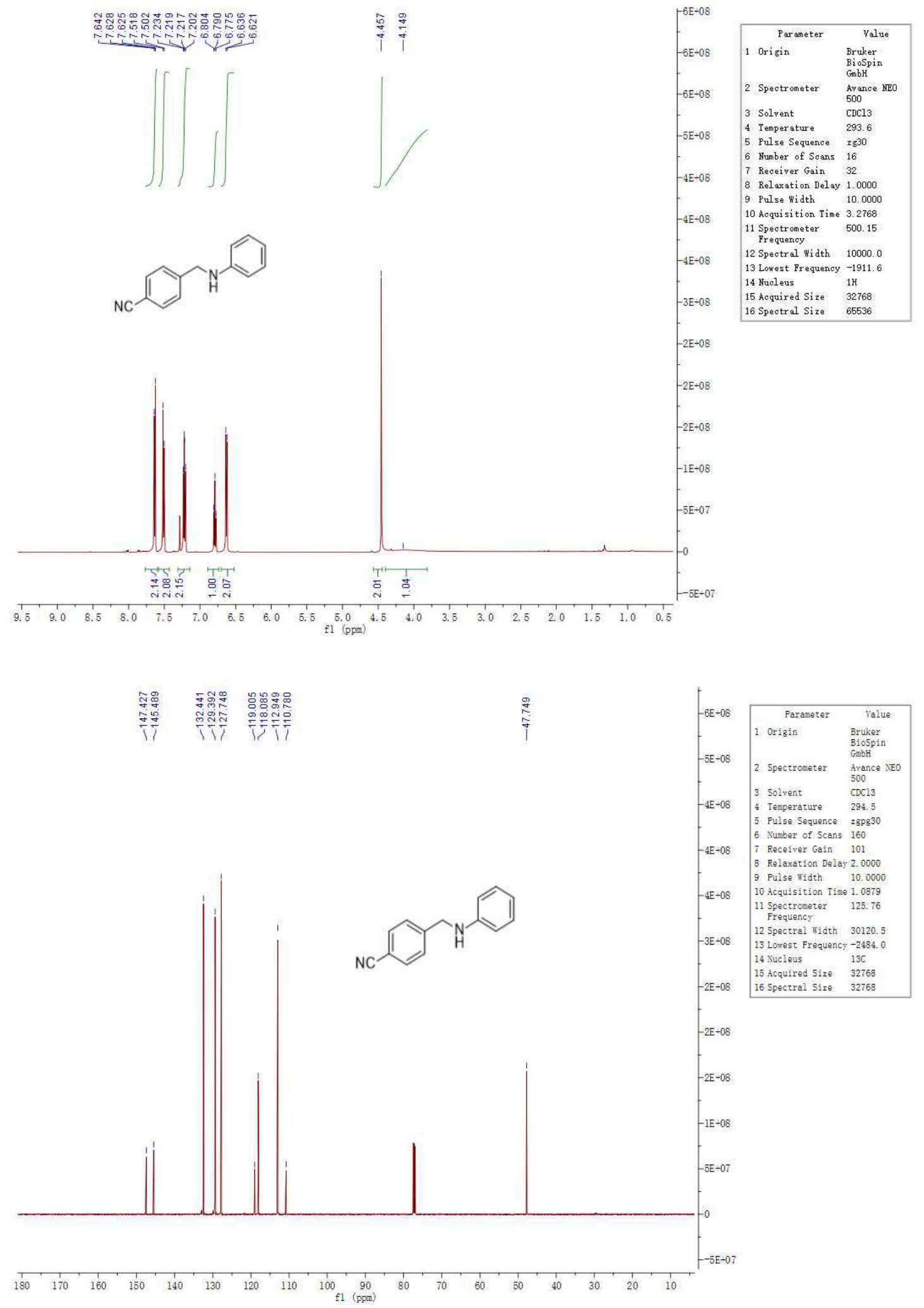
methyl 4-((p-tolylamino)methyl)benzoate (2s)
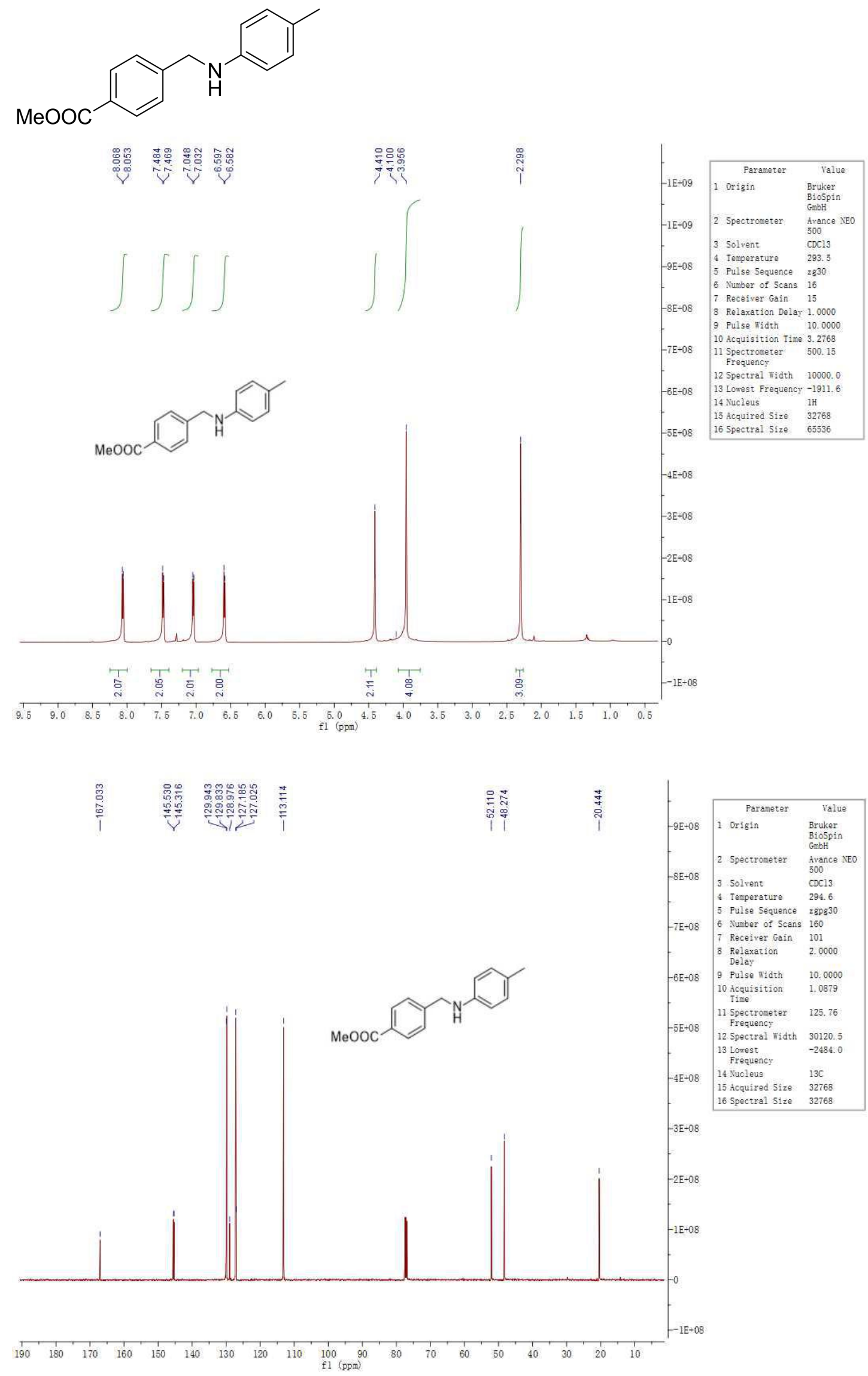
methyl 2-(benzhydrylamino)acetate (2t)<smiles>COC(=O)CNC(c1ccccc1)c1ccccc1</smiles>
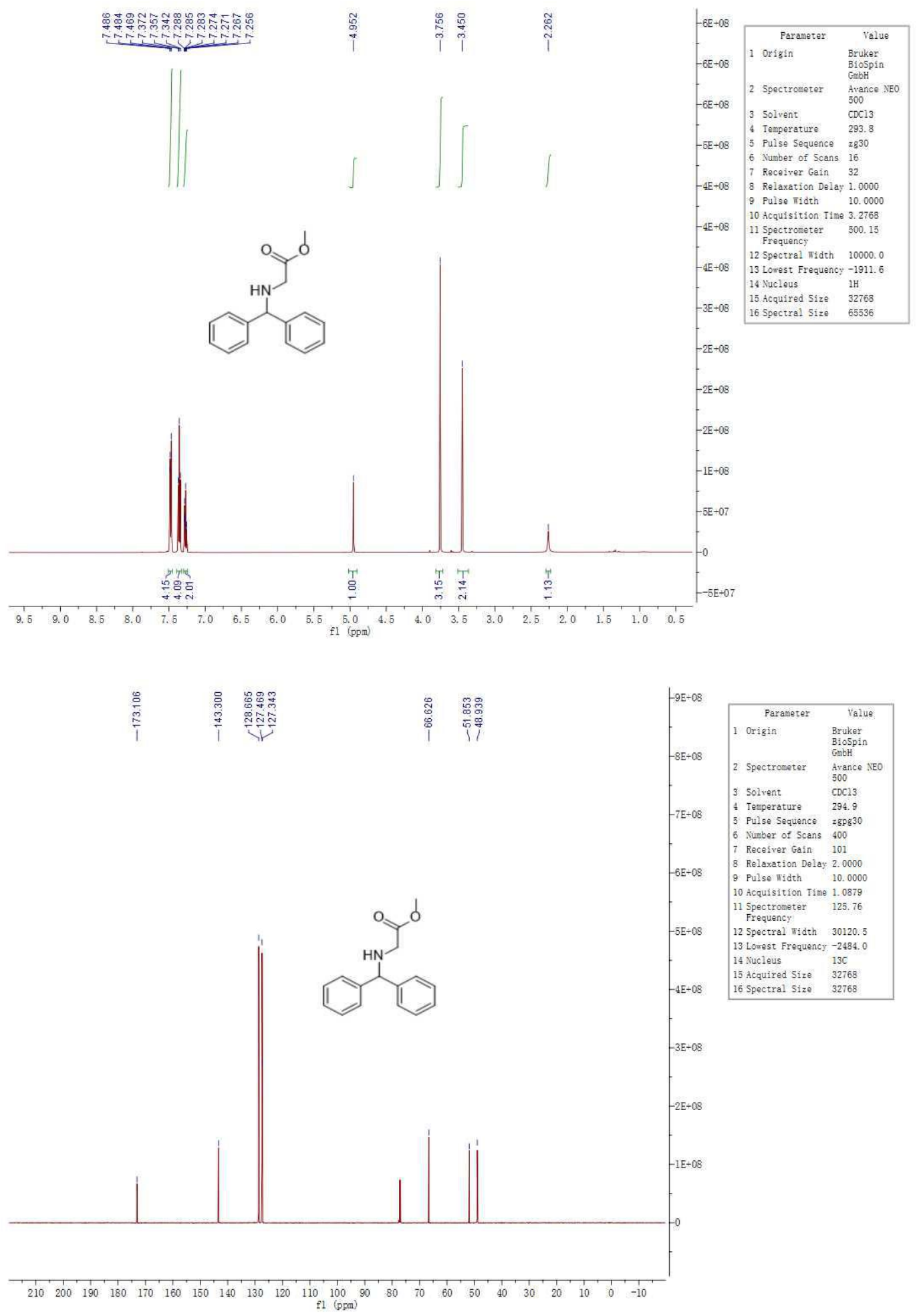
(E)-N-cinnamylaniline (2u)<smiles>C(=C/c1ccccc1)\CNc1ccccc1</smiles>
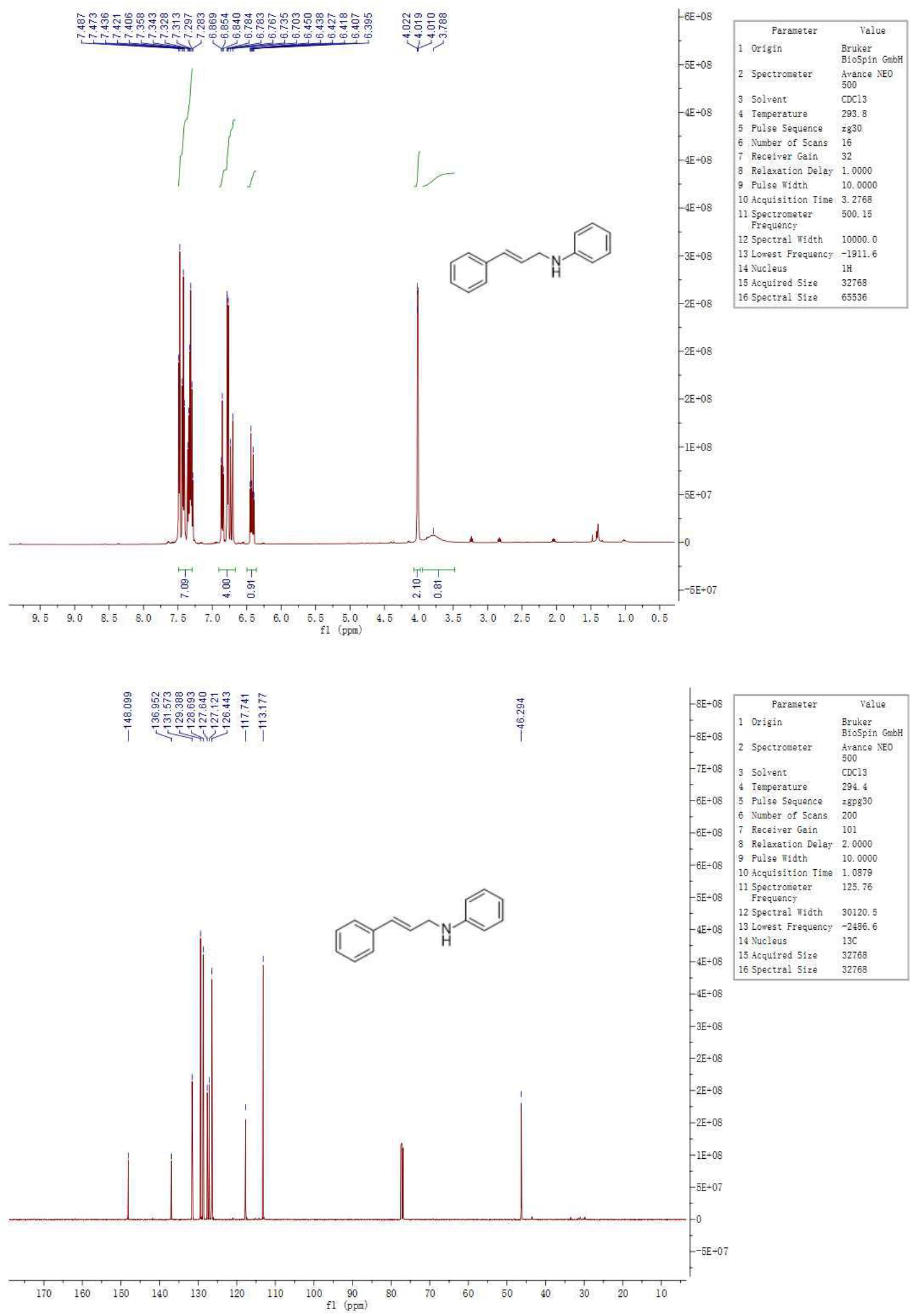
N-((5-methylthiophen-2-yl)methyl)aniline (2v)<smiles>Cc1ccc(CNc2ccccc2)s1</smiles>
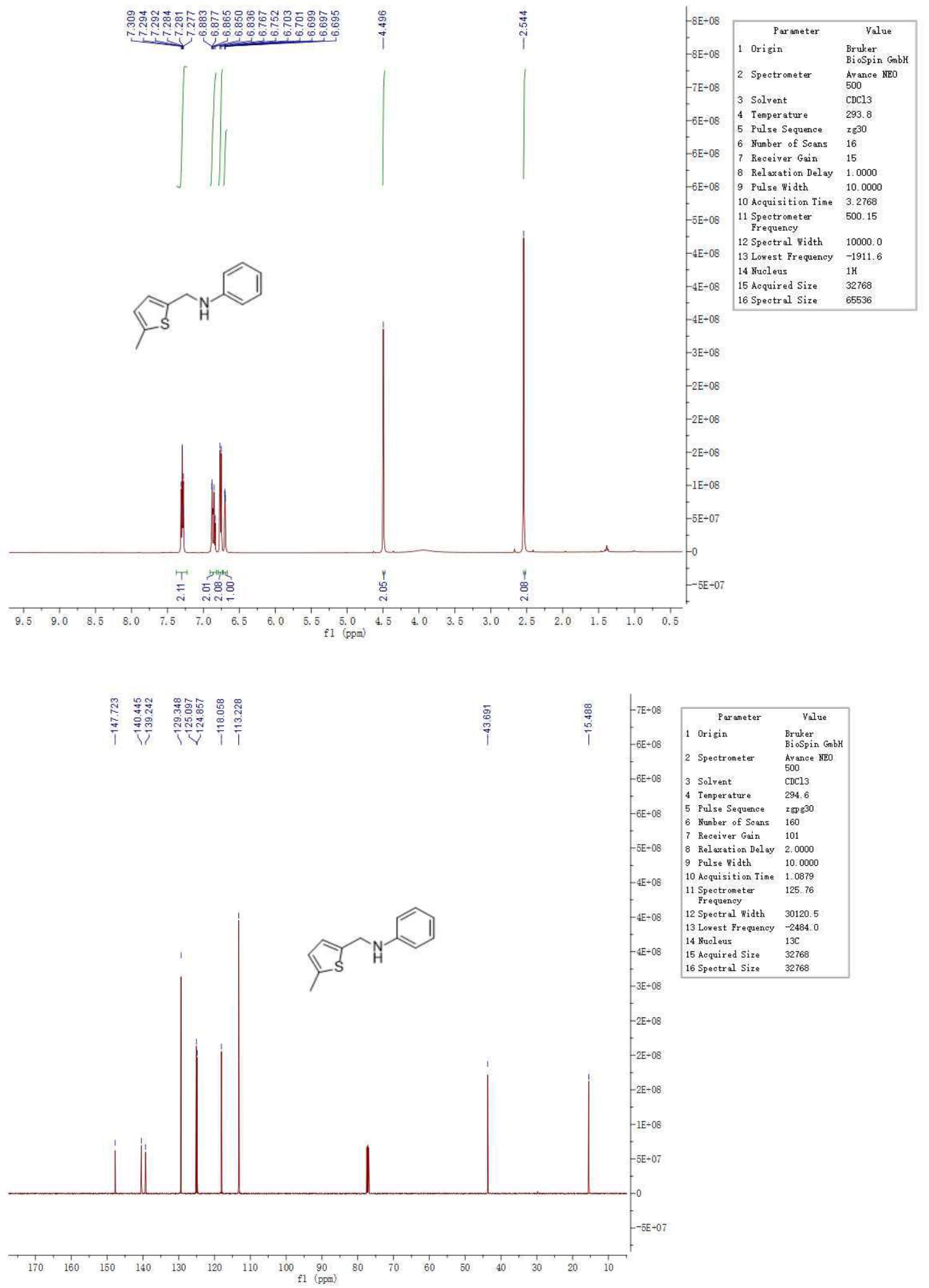
$N$-(naphthalen-2-ylmethyl)aniline (2w)<smiles>c1ccc(NCc2ccc3ccccc3c2)cc1</smiles>
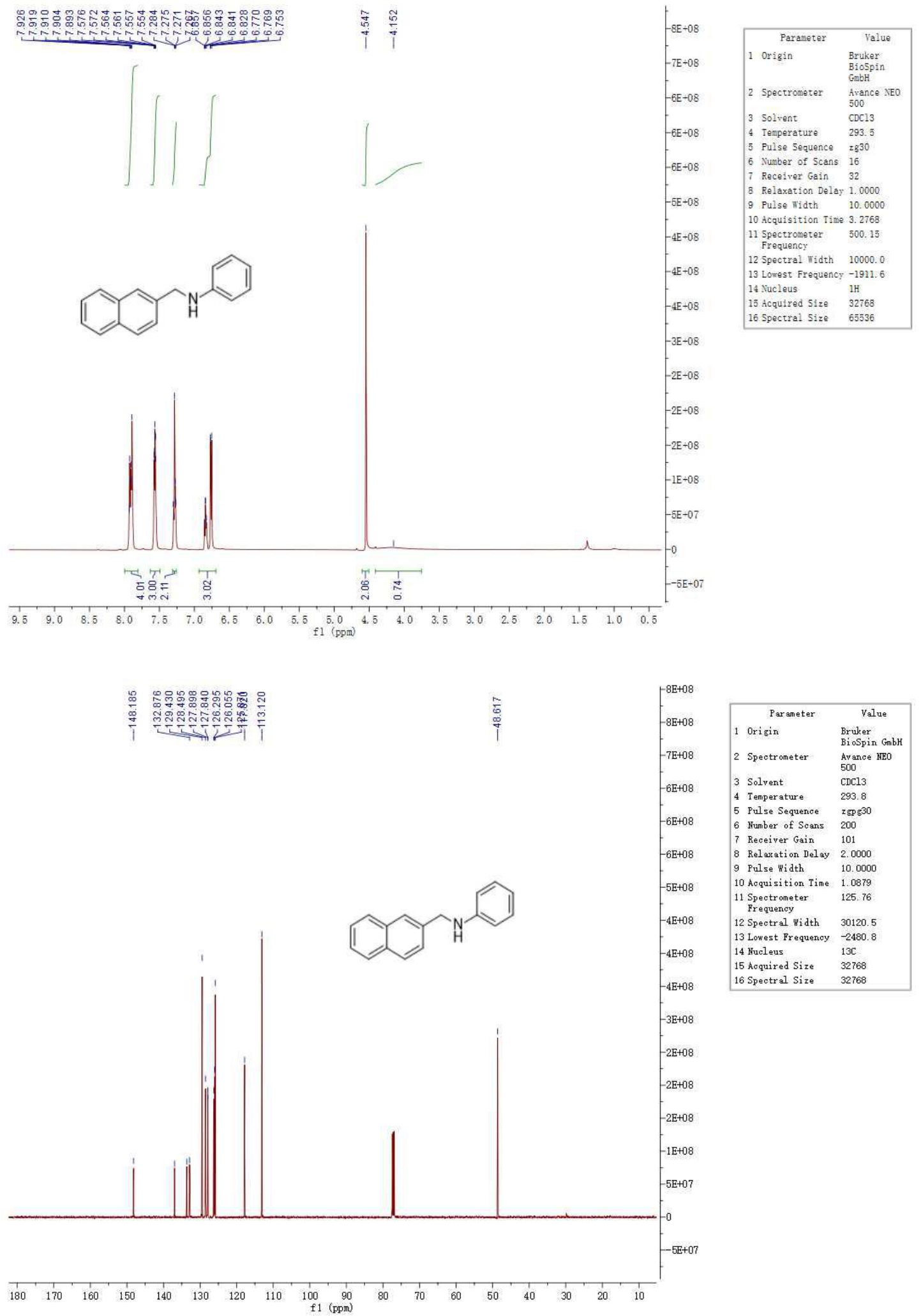
1-(benzo[d][1,3]dioxol-5-yl)-N-benzylmethanamine (2x)

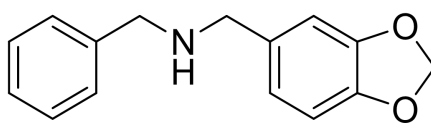

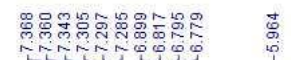

iniviving

ind<smiles>c1ccc(CNCc2ccc3c(c2)OCO3)cc1</smiles>

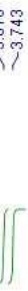

2 Spectrometer Avance NEO

3 Solvent CDC13

4 Temperature 293.1

5ulse 5equence $z 330$

5. Number of 5 cans 16

7 Receiver Gain 67

8 Relaxation Delay: 1.0000

9 Fulse Tidth 10,0000

10 Acquisition Time 3.2768

11 Spectrometar $\quad 500.15$

Frequency

12 spectral Tidth $\quad 10000.0$

13 Lowest Frequency -1911.

14 Nucleus $1 \mathrm{H}$

15 Acquired Size 32768

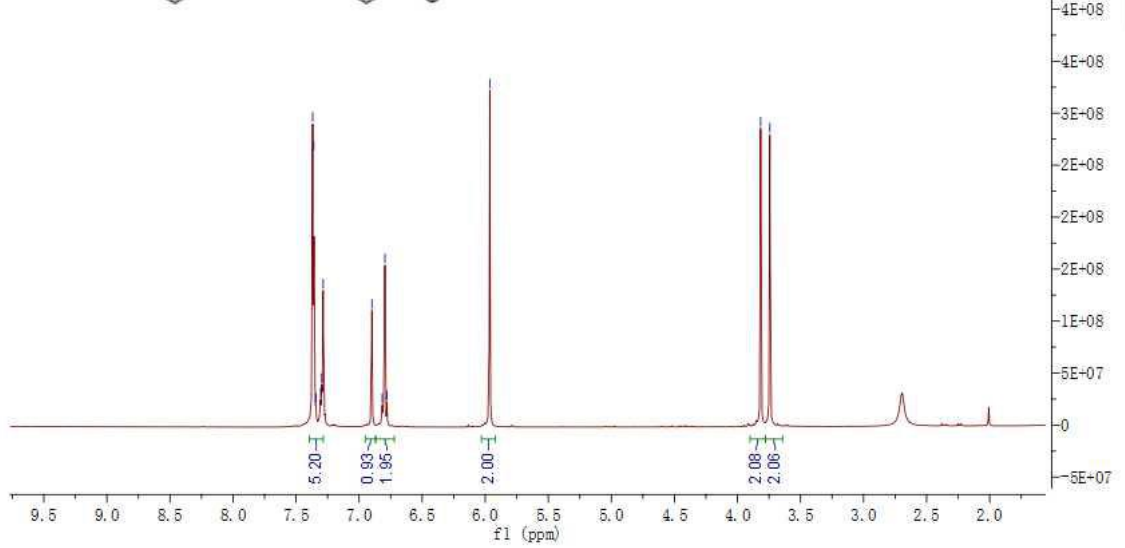

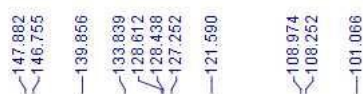

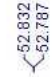

\begin{tabular}{|c|c|c|}
\hline \multirow{2}{*}{\multicolumn{2}{|c|}{$\begin{array}{l}\text { Parameter } \\
\text { Origin }\end{array}$}} & Yalue \\
\hline & & $\begin{array}{l}\text { Bruker } \\
\text { BioSpin } \\
\text { GmbH }\end{array}$ \\
\hline & Spectrometer & $\begin{array}{l}\text { Avance BEC } \\
500\end{array}$ \\
\hline 3 & Solvent & $\mathrm{CDCl3}$ \\
\hline 4 & Temperature & 294.7 \\
\hline & Pulse Sequence & z\&g 50 \\
\hline 6 & Number of Scans & 160 \\
\hline & Receiver Gain & 101 \\
\hline 8 & Relaxation Delay & 2.0000 \\
\hline 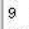 & Pulse Width & 10.0000 \\
\hline & Acquisition Time & 1.0879 \\
\hline & $\begin{array}{l}\text { Spectrometer } \\
\text { Frequency }\end{array}$ & 125.76 \\
\hline & Spectral width & 30120.5 \\
\hline & Lowest Frequency & -2484.0 \\
\hline & Hucleus & $13 C$ \\
\hline & Acquired Size & 32768 \\
\hline & Spectral Sire & 32768 \\
\hline
\end{tabular}
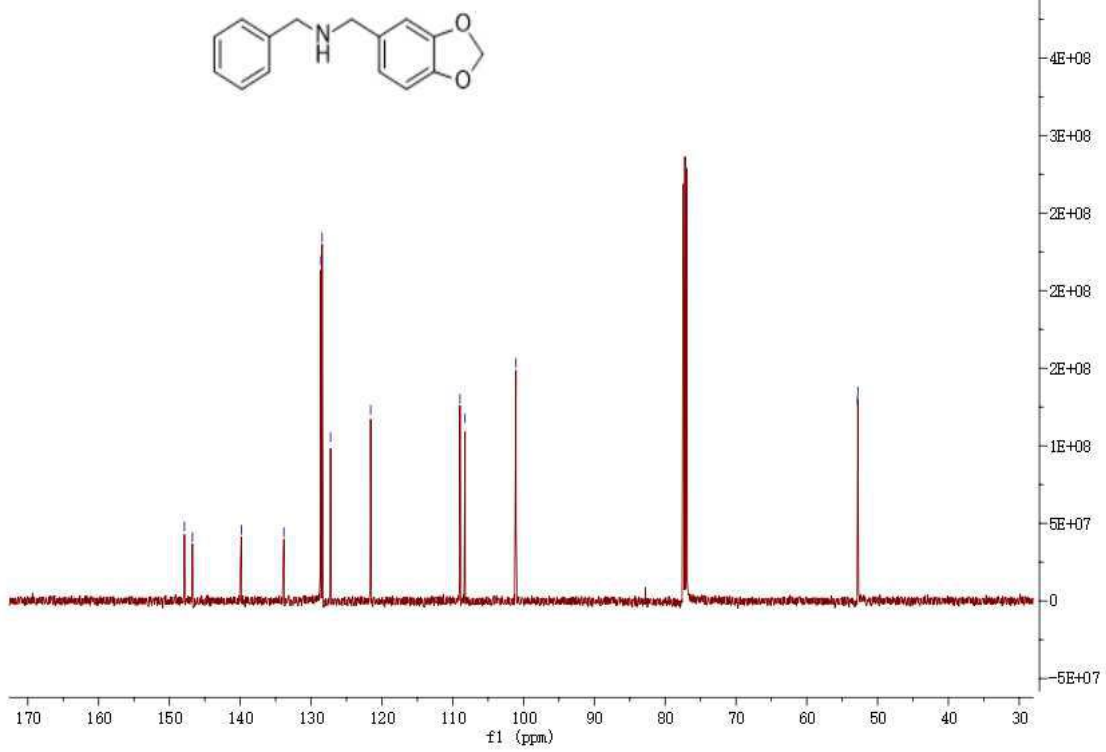
$N$-[(Ferrocenyl)methyl]aniline (2y)
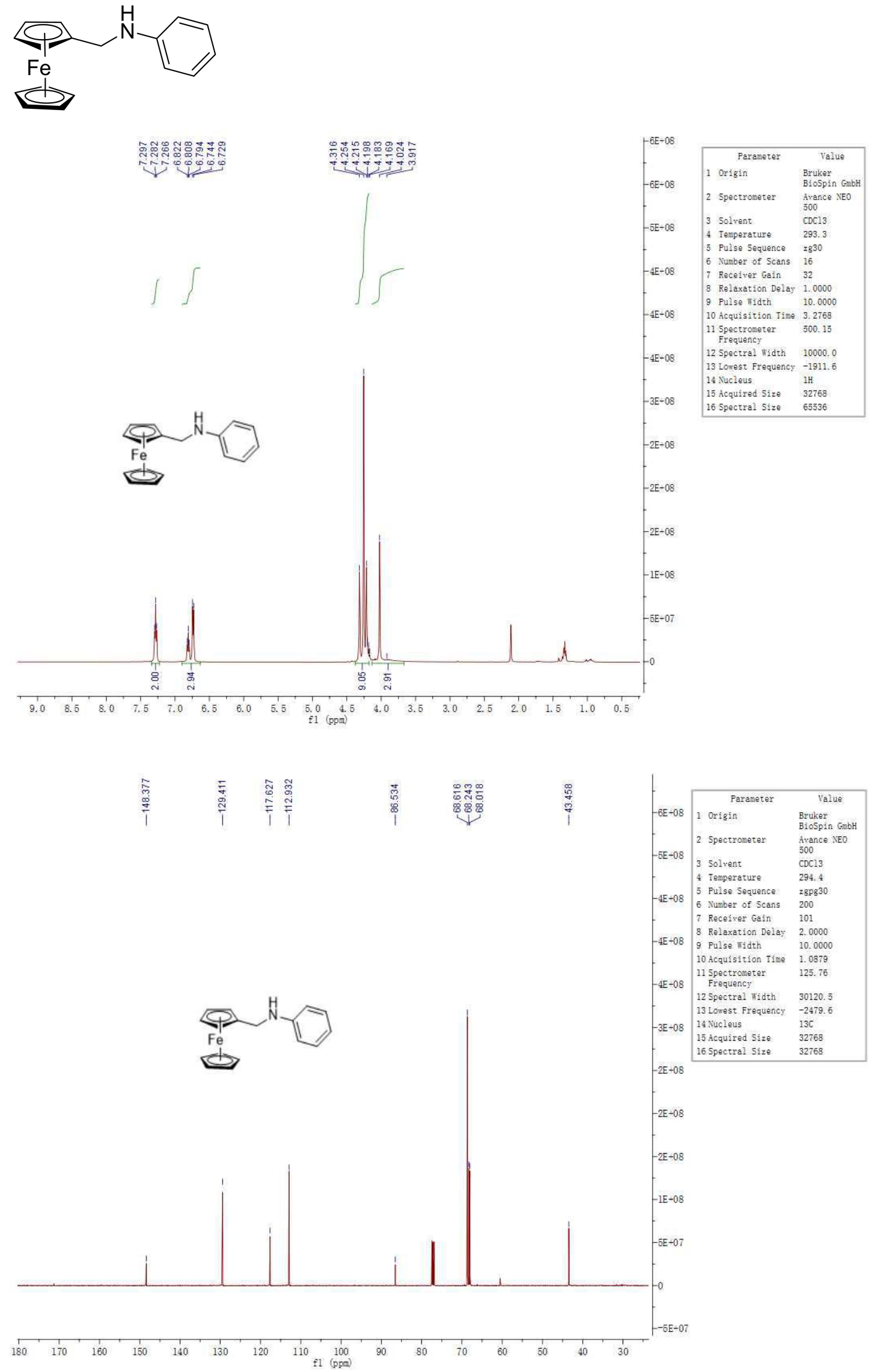
3-(4-(benzylamino)phenyl)-3-ethylpiperidine-2,6-dione (2z)<smiles>CCC1(c2ccc(NCc3ccccc3)cc2)CCC(=O)NC1=O</smiles>
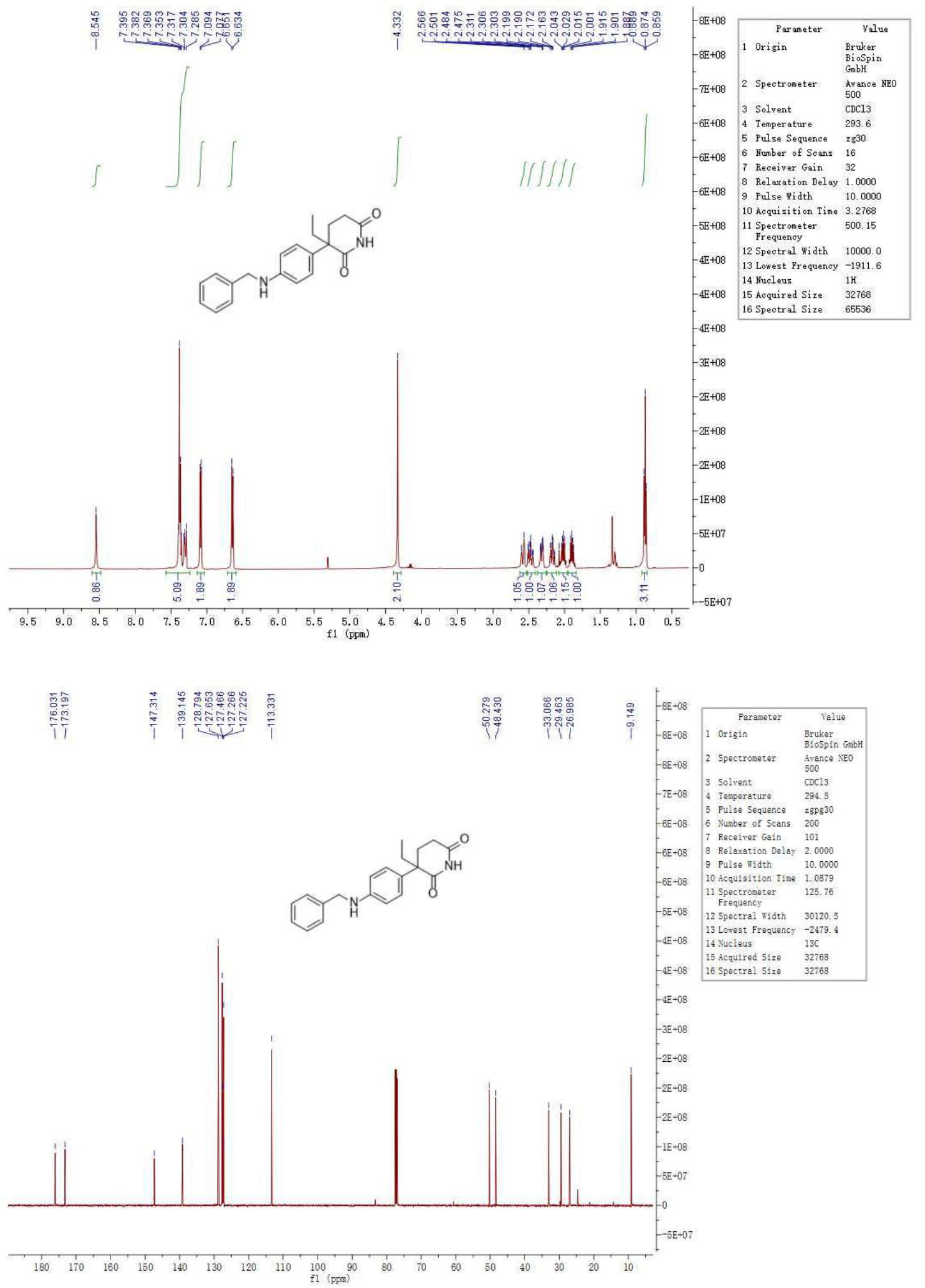